\title{
IRS-Assisted UAV Communications with Imperfect Phase Compensation
}

This paper was downloaded from TechRxiv (https://www.techrxiv.org).

\section{LICENSE}

CC BY 4.0

SUBMISSION DATE / POSTED DATE

28-10-2020 / 03-11-2020

\section{CITATION}

Al-Dweik, Arafat; Al-Jarrah, MOHAMMAD AHMAD; Alsusa, Emad; Alouini, Mohamed-Slim; Iraqi, Youssef (2020): IRS-Assisted UAV Communications with Imperfect Phase Compensation. TechRxiv. Preprint. https://doi.org/10.36227/techrxiv.13153211.v1

$\mathrm{DOI}$

10.36227/techrxiv.13153211.v1 


\title{
IRS-Assisted UAV Communications with Imperfect Phase Compensation
}

\author{
M. Al-Jarrah, Member, IEEE, A. Al-Dweik, Senior Member, IEEE, E. Alsusa, Senior Member, IEEE, \\ Y. Iraqi, Senior Member, IEEE, and M.-S. Alouini, Fellow, IEEE
}

\begin{abstract}
This work presents a performance analysis on unmanned aerial vehicles (UAVs) assisted wireless communications systems, where one of the UAVs supports intelligent reflecting surfaces (IRS). As the estimation and compensation of the end-to-end phase for each propagation path is prone to errors, imperfect phase compensation at the IRS is taken into consideration. The performance is derived in terms of symbol error rate (SER) and outage probability, where the phase error is modeled using the von Mises distribution. The analysis utilises the Sinusoidal Addition Theorem (SAT) when the number of reflectors $L \leq 3$, and the Central Limit Theorem (CLT) when $L \geq 4$. The achieved results show that accurate phase estimation and compensation is critical for IRS based systems, particularly for a small number of reflecting elements. For example, the SER at $10^{-3}$ degrades by about $5 \mathrm{~dB}$ when the von Mises concentration parameter $\kappa=2$ and $L=30$, but the degradation for the same $\kappa$ surges to $25 \mathrm{~dB}$ when $L=2$. The air-to-air (A2A) channel for each propagation path is modeled as a single dominant line-of-sight (LoS) component, and the results are compared to the Rician channel model. The obtained results reveal that the considered A2A model can be used to accurately represent the A2A channel with Rician fading.
\end{abstract}

\section{Index Terms}

Bit error rate (BER), outage probability, Rician fading, intelligent reflecting surfaces (IRS), imperfect phase estimation, sinusoidal addition theorem (SAT), unmanned aerial vehicle (UAV), flying network, von Mises density, 6G.

\section{INTRODUCTION}

Intelligent reflecting surfaces (IRSs), also called metasurfaces, is an emerging technology that has recently received extensive attention [1]-[13]. The main aim of IRS is controlling the propagation medium to improve the quality of wireless signals by increasing their total energy. The IRS technology

M. A. Al-Jarrah and E. Alsusa are with the School of Electrical and Electronic Engineering, University of Manchester, Manchester M13 9PL, U.K. (Email: \{mohammad.al-jarrah, E.alsusa\}@manchester.ac.uk).

A. Al-Dweik and Y. Iraqi are with the Center for Cyber Physical Systems, Khalifa University, Abu Dhabi, UAE. (E-mail: \{arafat.dweik, youssef.iraqi\}@ku.ac.ae). A. Al-Dweik is also with the Department of Electrical and Computer Engineering, Western University, London, ON, Canada. (E-mail: dweik@fulbrightmail.org).

M.-S. Alouini is with King Abdullah University of Science and Technology (KAUST), Thuwal, Makkah Province, Kingdom of Saudi Arabia, (E-mail: slim.alouini@kaust.edu.sa). 
is expected to play a significant role in future wireless networks, such as sixth generation (6G), because of its positive impact on energy and spectral efficiency. IRS consist of a large number of passive antenna elements that can introduce phase-shifts to wireless signals before reflecting them to their destination. For efficient transmission, multiple reflectors are used for a certain destination, and the introduced phase shifts are selected such that the reflected signals add coherently in the channel. As a result, the signalto-noise ratio (SNR) increases considerably, which allows using high modulation orders to improve the spectral efficiency.

Likewise, the use of unmanned aerial vehicles (UAVs) as flying networks has recently attracted a substantial attention in both academic and industrial sectors. Because of their autonomy, flexibility and cost efficiency, there has been a significant growth in the deployment of UAVs in many applications including surveillance, localization and tracking, remote sensing, search and rescue missions, aerial imaging, and military applications. In addition, UAVs can be integrated with base stations (BS) to construct cost and energy efficient flying BSs. These flying BSs can provide integrated access and backhaul (IAB) solutions with significantly improved coverage, capacity and connectivity. Furthermore, UAV based IAB can assist the terrestrial cellular network that may suffer from congestion due to extremely high traffic or physical failure due to emergencies such as storms and earthquakes [14]-[20].

Although IRS may lead to significant SNR gain, such gain is highly dependent on the system capability to accurately estimate and compensate the end-to-end phase for each IRS element, which is one of the main challenges for such technology. Therefore, channel estimation, phase shift design, and performance evaluation with imperfect phase have received extensive attention [13], [21]-[29]. The phase estimation and compensation problem becomes more critical when IRS is integrated with flying networks. In such contexts, UAV assisted communications in urban areas may employ IRSs to improve the signal quality in the absence of line-of-sight $(\mathrm{LoS})$ connectivity between certain UAVs due to Skyscrapers [12], [30]-[39]. Nevertheless, UAVs are typically moving causing fast variation to the channel phase, which complicates the overall phase processing. Channel variations are expected even when the UAV is hovering due to the UAV wobbling [40], and thus, the coherence time of the channel will be generally short. Therefore, the IRS might be provided with outdated phase information. Hence, performance analysis of IRS based systems while considering imperfect co-phasing process is crucial. 


\section{A. Related Work}

existing research work on IRS has covered a broad range of topics such as, but not limited to, power and energy optimization [1], [8], [13], [33], physical layer security [7], resource allocation with non orthogonal multiple access (NOMA) [9], full duplex cognitive radio [5], and symbol-level precoding [11]. The integration of IRS and simultaneous wireless information and power transfer (SWIPT) is considered in [4]. Gao et al. considered the design of distributed IRSs with passive reflecting beamforming that exploits statistical channel state information (CSI) and analyzed the ergodic achievable rate. Xie $e t$ al. [3] formulated and solved a joint optimization problem for the coordinated transmit and reflective beamforming for maximizing the minimum weighted received signal-to-interference-plus-noise ratio (SINR) at users subject to transmit power constraints.

The integration of IRSs and UAVs has also been considered in the literature. For example, Lu et al. [12] proposed deploying flying platforms such as balloons or UAVs equipped with IRS to serve terrestrial users. The presented results show that flying IRS has an extra degree of freedom because of the capability of relocating the IRS to optimize certain system parameters such as maximizing the SNR. Moreover, it is shown that flying IRSs require less number of elements to achieve a certain gain as compared to terrestrial IRSs. Jiao et al. [30] investigated the design of a NOMA-based IRS-UAV system to maximize the rate of the near user while guaranteeing the target rate of the far user by optimizing the UAV location, transmit beamforming and phase shift of IRS. Ma et al. [31] used the IRS to direct the signal to the UAV to increase its received signal strength. The obtained results show that significant signal improvement can be obtained using a small number of reflectors given that the location of the IRSs and phase of the reflected signals are optimized. Ge et al. [32] considered a system where a single UAV transmits to multiple terrestrial IRSs. The work focused on the optimal design of beamforming at the UAV, IRSs and the UAV's trajectory to maximize the received power at the ground users. Mohamed and Aïssa [33] considered the downlink of a multi-antenna BS that communicates to a single antenna user via an IRS-UAV platform. The work evaluates the advantage of the IRS to maximize the total energy efficiency of the system by jointly optimizing the beamforming vector at the BS and the phase shifts matrix of the IRS. Various optimization techniques under the assumption of perfect CSI. Several other articles have considered integrated IRS-UAV [34]-[39] to minimize the transmit power, maximize the SNR, maximize the spectral efficiency, or maximize the sum rate. Nevertheless, they did not consider the error or outage probability analysis, or the impact of imperfect phases estimation and control process. 
The impact of the phase modeling, estimation and compensation has been considered by Abeywickrama et al. [13], who proposed a more practical phase model that considers the correlation between the phase and amplitude of the individual reflected signals. The authors formulated an optimization problem to minimize the total transmit power by jointly designing the transmit and IRS beamforming. Although the phase model is interesting, the phase estimation and control processes are considered perfect. Moreover, the error and outage probabilities are not considered. The authors in [21] considered the outage probability of IRS-NOMA with coherent and random phase processing. For the coherent case, the phase estimation and compensation were considered ideal, while the random phase was considered uniform and discrete. The presented outage probability results show that IRS can still provide some gain even when the phase is random. The work does not consider the error probability nor the coherent scenario with phase error. The CSI estimation and discrete phase model are considered in [22], where the presented results, in terms of the achievable rate, demonstrate the significant impact of using a discrete phase. $\mathrm{Hu}$ et al. [23] considered the imperfect phase scenario by introducing user location uncertainty. The objective of this work is to minimize the transmit power subject to quality of service (QoS) constraint. CSI estimation has also been considered in [24]-[29], though the focus of these works is mostly on evaluating the CSI estimates accuracy, rather than evaluating its impact on the system performance.

The error probability analysis of IRS based systems has been considered in [41]-[46]. Nevertheless, the only work that considered the bit error rate with imperfect phase estimation is [47], where the phase error and fading coefficients are modeled as von Mises and Nakagami- $m$ distributions, respectively. However, the presented derivations in [47, Eq. 13, Eq. 14] actually correspond to the case of uniform phase distribution [48], [49]. Consequently, the analysis is applicable to the random phase scenario considered in [21].

\section{B. Motivation and Main Contributions}

As can be noted from the surveyed literature, integrating IRS with flying networks using UAVs has a strong potential to improve UAVs connectivity in urban areas. The extra degree of freedom that UAVs have can enable optimizing the IRS link by selecting the most suitable placement for the IRSUAV. Nevertheless, achieving the ultimate gain using IRS is highly dependent on the reliability of the phase estimates and co-phasing processes. Practically speaking, both operations are not perfect, and thus, the ultimate gain promised by the IRS technology may not be guaranteed, which is particularly 
TABLE I: Nomenclature.

\begin{tabular}{|c|c||c|c|}
\hline Symbol & Definition & Symbol & Definition \\
\hline \hline$s$ & Complex data symbol & $\psi$ & Channel phase, Tx $\rightarrow$ IRS \\
$r$ & Received passband signal at IRS & $y$ & Received passband signal at Rx \\
$T_{s}$ & Symbol duration & $\hbar_{i}$ & Channel attenuation, IRS $\rightarrow$ Rx \\
$p(t)$ & Pulse shape & $\phi_{i}$ & Channel phase, IRS $\rightarrow$ Rx \\
$L$ & Number of IRS elements & $z$ & AWGN at Rx \\
$f_{\mathrm{c}}$ & Carrier frequency & $\sigma_{z}^{2}$ & AWGN variance \\
$a$ & Amplitude of data symbol, $|s|$ & $\zeta_{L}$ & Signal phase at Rx \\
$\varphi$ & Phase of data symbol, tan ${ }^{-1}\left(\frac{\Im\{(\ell)\}}{\Re\{s(\ell)\}}\right)$ & $B_{L}$ & Signal amplitude at Rx \\
$\tau_{i}$ & Time delay of the $i$ & $d$ & Decision variable at Rx \\
$h$ & Channel attenuation, Tx $\rightarrow \operatorname{IRS}$ & $\mu$ & Phase error mean \\
$\tau$ & Channel delay, Tx $\rightarrow \operatorname{IRS}$ & $\kappa$ & Phase error shape parameter \\
$P_{\mathrm{e}}$ & Conditional SER & $I_{0}(\cdot)$ & Modified Bessel function \\
$\mathcal{K}$ & Rician fading factor & $\bar{P}_{\mathrm{e}}$ & Average SER \\
\hline
\end{tabular}

critical for IRS-UAV configurations. Therefore, this work analyzes the performance of IRS assisted UAV communications under a more realistic scenario, where the phase estimation and co-phasing processes are imperfect. The phase error is modeled using the von Mises distribution, and the channels are considered to have a dominant LoS component. The performance is evaluated in terms of symbol error rate (SER) and outage probability, where exact closed-form expressions are derived for a small number of reflecting elements, and accurate approximations are derived for a large number of reflectors. The obtained results show that the gain achieved using IRSs depends strongly on the reliability of the co-phasing process, particularly when the number of reflectors is small. For a large number of reflectors, the system sensitivity to the co-phasing process decreases significantly.

\section{Notations}

For the readers' convenience, the nomenclature and main symbol definitions are given in Tables I and II, respectively.

\section{Paper Organization}

The rest of the paper is organized as follows. Sec. II presents the system and channel models. Sec. III presents the derivation of the signal envelope distribution for different number of reflectors. Secs. IV and $\mathrm{V}$ present the SER and outage probability analysis. Numerical and simulation results are presented in Sec. VI, and finally the paper is concluded in Sec. VII. 
TABLE II: Frequently used definitions.

\begin{tabular}{|l||l|}
\hline$\alpha=j\left(b_{2}^{2}-A_{1}^{2}-A_{2}^{2}\right), j=\sqrt{-1}$ & $\dot{\alpha}=j\left(b_{3}^{2}-b_{2}^{2}-A_{3}^{2}\right)$ \\
$\tilde{\alpha}=-j \alpha$ & $\check{\alpha}=b_{3}^{2}-b_{2}^{2}-A_{3}^{2}$ \\
$\beta=-2\left|A_{1} A_{2}\right|$ & $\dot{\beta}=-2\left|A_{3} b_{2}\right|$ \\
$\mathcal{B}\left(b_{2}\right) \triangleq \mathcal{B}=\sqrt{\alpha^{2}+\beta^{2}}$ & $\dot{\mathcal{B}}\left(b_{3}\right) \triangleq \dot{\mathcal{B}}=\sqrt{\dot{\alpha}^{2}+\dot{\beta}^{2}}$ \\
$K=\frac{\kappa_{2}}{2 A_{1} A_{2}}$ & $\tilde{K}=\frac{\kappa_{3}}{2 A_{3} b_{2}}$ \\
$v=-K\left(\sin \left(\epsilon_{1}\right)+j \cos \left(\epsilon_{1}\right)\right)$ & $\dot{v}=-\bar{K}\left(\sin \left(\zeta_{2}\right)+j \cos \left(\zeta_{2}\right)\right)$ \\
$\theta_{i}=\psi_{i}+\phi_{i}$ & $\epsilon_{i}=\hat{\theta}_{i}-\theta_{i}$ \\
$\lambda=K \sin \left(\epsilon_{1}\right)$ & $\check{\lambda}=\tilde{K} \sin \left(\zeta_{2}\right)$ \\
$\mathcal{B}_{2}\left(b_{2}\right) \triangleq \mathcal{B}_{2}=\frac{\tilde{\alpha}}{2 A_{1} A_{2}}$ & $\mathcal{B}_{3}\left(b_{3}\right) \triangleq \mathcal{B}_{3}=\frac{\check{\alpha}}{2 A_{3} b_{2}}$ \\
$\mathcal{A}_{1}=2 \sqrt{A_{1} A_{2}}$ & $I_{0}^{(2)}(\boldsymbol{\kappa})=I_{0}\left(\kappa_{1}\right) I_{0}\left(\kappa_{2}\right)$ \\
$\mathcal{A}_{2}=\left(A_{1}-A_{2}\right)^{2}$ & $I_{0}^{(3)}(\boldsymbol{\kappa})=I_{0}\left(\kappa_{1}\right) I_{0}\left(\kappa_{2}\right) I_{0}\left(\kappa_{3}\right)$ \\
$\mathcal{A}_{3}=\left(A_{1}+A_{2}\right)^{2}$ & $A_{i}=g_{i} \hbar_{i} h_{i}$ \\
\hline
\end{tabular}

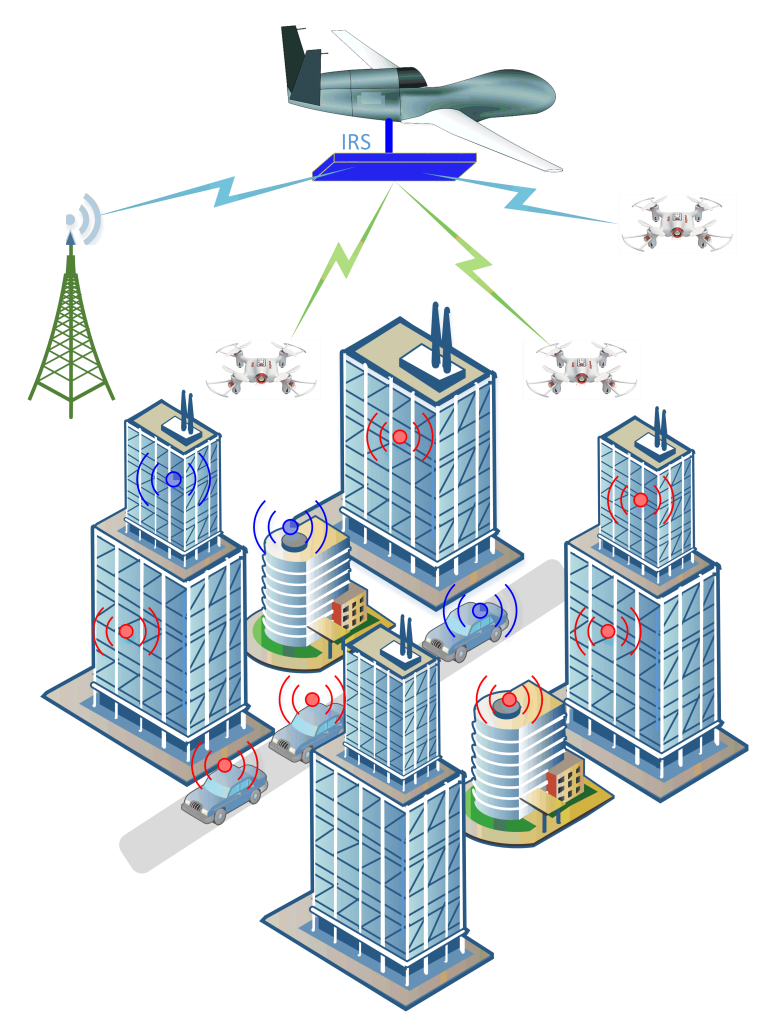

Fig. 1: IRS assisted flying networks. 


\section{System AND Channel Models}

This work considers an IRS-assisted UAV communications as shown in Fig. 1. In flat fading channels, the passband signal arriving at the $i$ th reflecting element can be written as

$$
\begin{aligned}
r_{i}(t) & =h_{i} s \cos \left[\omega_{\mathrm{c}} t-\psi_{i}\right] \\
& =h_{i} a \cos \left[\omega_{\mathrm{c}} t+\varphi-\psi_{i}\right], i=[1,2, \ldots, L]
\end{aligned}
$$

where $s$ is the complex information symbol, $s=a \mathrm{e}^{j \varphi}, \omega_{\mathrm{c}}=2 \pi f_{\mathrm{c}}, f_{\mathrm{c}}$ is the carrier frequency, $h_{i}$ is the channel fading coefficient between the transmitter and the $i$ th reflecting element. The phases $\psi_{i} \forall i$ are typically modeled as mutually independent and identically distributed (i.i.d.) random variables that are uniformly distributed over $[-\pi, \pi)$ [50]. Each IRS element shifts the signal phase by a value $\theta_{i}$ and attenuates the signal by a factor $g_{i}$. Therefore, using the same assumptions for the signal arriving at the IRS, the reflected $L$ signals arriving from the IRS at the receiver can be written as

$$
y(t)=\sum_{i=1}^{L} g_{i} \hbar_{i} h_{i} a \cos \left[\omega t+\varphi-\psi_{i}-\phi_{i}+\theta_{i}\right]+z(t)
$$

where $\hbar_{i}$ and $\phi_{i}$ are attenuation and phase shift caused by the channel between the $i$ th IRS element and the receiver, $z(t)$ is the additive white Gaussian noise (AWGN). To maximize the received SNR $\theta_{i}$ is selected such that $\theta_{i}=\psi_{i}+\phi_{i}$, and then $y(t)$ can be written as

$$
\begin{aligned}
y(t) & =\left(\sum_{i=1}^{L} g_{i} \hbar_{i} h_{i} a\right) \cos [\omega t+\varphi]+z(t) . \\
& =B_{L} \cos \left[\omega_{c} t+\varphi\right]+z(t) .
\end{aligned}
$$

However, it is practically infeasible to estimate and compensate the phases $\psi_{i}$ and $\phi_{i}$. Therefore, $y(t)$ with imperfect phase estimation and compensation should be written as

$$
y(t)=\sum_{i=1}^{L} g_{i} \hbar_{i} h_{i} a \cos \left[\omega t+\varphi-\psi_{i}-\phi_{i}+\hat{\theta}_{i}\right]+z(t) .
$$


where $\hat{\theta}_{i}=\hat{\psi}_{i}+\hat{\phi}_{i}, \hat{\psi}_{i}$ and $\hat{\phi}_{i}$ are the estimated and compensated versions of $\psi_{i}$ and $\phi_{i}$, respectively. Thus,

$$
\begin{aligned}
y(t) & =a \sum_{i=1}^{L} g_{i} \hbar_{i} h_{i} \cos \left[\omega t+\varphi+\epsilon_{i}\right]+z(t) \\
& =a \sum_{i=1}^{L} A_{i} \cos \left[\omega t+\varphi+\epsilon_{i}\right]+z(t)
\end{aligned}
$$

where $\epsilon_{i}=\hat{\theta}_{i}-\theta_{i}, g_{i} \hbar_{i} h_{i} \triangleq A_{i} \in(-\infty, \infty)$, and $\epsilon_{i} \in[-\pi, \pi]$. Using the Sinusoidal Addition Theorem (SAT) [48], [49] we obtain

$$
y(t)=a B_{L} \cos \left(\omega t+\varphi+\phi_{L}\right), t \geq 0
$$

where

$$
\begin{gathered}
B_{L}^{2}=\|\mathbf{A}\|^{2}+2 \sum_{L \geq j>k \geq 1} A_{j} A_{k} \cos \left(\epsilon_{j}-\epsilon_{k}\right) \\
\zeta_{L}=\tan ^{-1}\left[\frac{\sum_{i=1}^{L} A_{i} \sin \left(\epsilon_{i}\right)}{\sum_{i=1}^{L} A_{i} \cos \left(\epsilon_{i}\right)}\right]
\end{gathered}
$$

and $\|\cdot\|$ is the Euclidian norm.

At the receiver, the carrier signal will be removed and the data symbol during the $\ell$ th signaling period can expressed as

$$
\begin{aligned}
d & =\frac{1}{T_{s}} \int_{0}^{T_{s}} 2 y(t) \mathrm{e}^{-j\left(\omega t+\hat{\zeta}_{L}\right)} d t \\
& =a B_{L} \mathrm{e}^{j\left(\varphi+\zeta_{L}-\hat{\zeta}_{L}\right)}+z
\end{aligned}
$$

where $\hat{\zeta}_{L}$ is an estimate of the accumulated phase offset $\zeta_{L}$, and $z \sim \mathcal{C N}\left(0,2 \sigma_{z}^{2}\right)$ is the AWGN. In slow fading channels, $\zeta_{L}$ can be estimated and compensated accurately, and thus $\hat{\zeta}_{L} \approx \zeta_{L}$. By noting that $a e^{j \varphi}=s$, then

$$
d=B_{L} s+z
$$

The SER can be derived as,

$$
\bar{P}_{\mathrm{e}}=\int_{0}^{\infty}\left(P_{\mathrm{e}} \mid b_{L}\right) f_{B_{L}}\left(b_{L}\right) d b_{L}
$$

where $f_{B_{L}}\left(b_{L}\right)$ is the probability density function (PDF) of the signal envelope $B_{L}$ and $P_{\mathrm{e}} \mid b_{L}$ is the conditional SER given $b_{L}$. The elements of $\mathbf{A}_{L}=\left[A_{1}, \ldots, A_{L}\right]$ depend on the channel model. For air-to-air channels (A2A), the signal typically has a strong LoS and some reflected components, and 
thus, such channels can be modeled using the Rician fading [51]-[57]. However, as the measurements indicated, the Rice factor $\mathcal{K}$ for $\mathrm{A} 2 \mathrm{~A}$ channel is about $20 \mathrm{~dB}$, and the received signal power may remain constant for long time periods [51]-[57]. Consequently, the channel coefficients $A_{1}, A_{2}, \ldots, A_{L}$ are not suffering from small scale fading, and the large scale fading is dominated by the free space path loss. Nevertheless, the obtained results in Sec. VI show that the constant fading coefficients model can be used to closely approximate the Rician fading channel with high $\mathcal{K}$ values.

\section{PDF OF THE SIGNAL ENVELOP $B_{L}$}

For a given values of $A_{1}, A_{2}, \ldots, A_{L}$, the PDF of $B_{L}$ can be computed as [48], [49]

$$
f_{B_{L}}\left(b_{L}\right)=\frac{b_{L}}{\pi} \int_{-\infty}^{\infty} \mathrm{e}^{-j b_{L}^{2} t} \int_{-\pi}^{\pi} \mathrm{e}^{j t\left\{b_{L-1}^{2}+A_{L}^{2}+2 A_{L} b_{L-1} \cos \left(\epsilon_{L}-\zeta_{L-1}\right)\right\}} \times f_{\epsilon}(\boldsymbol{\epsilon}) d \boldsymbol{\epsilon} d t
$$

where $\boldsymbol{\epsilon}=\left[\epsilon_{1}, \epsilon_{2}, \ldots, \epsilon_{L}\right]$ are the phase errors for the $L$ reflecting elements. The PDF $f_{B_{L}}\left(b_{L}\right)$ is derived in [48], [49] for the uniform phase $\epsilon_{i} \sim \mathcal{U}[-\pi, \pi] \forall i$, and has been used to model fading, interference and jamming in wireless systems [50], [58], [59]. However, when the SAT is used to model phase estimation errors, the uniform phase model is not applicable for such scenarios [60], because the phase error generally follows the von Mises distribution with mean $\mu$ and shape parameter $\kappa$ [61],

$$
f_{\epsilon_{i}}\left(\epsilon_{i}\right)=\frac{\mathrm{e}^{\kappa_{i} \cos \left(\epsilon_{i}-\mu_{i}\right)}}{2 \pi I_{0}\left(\kappa_{i}\right)}
$$

where $I_{q}($.$) is the modified Bessel function of the first kind and order q$. As can be noted form (13), the uniform PDF is a special case of the von Mises PDF with $\kappa=0$, which corresponds to the worst case phase error. For large values of $\kappa$, the PDF becomes concentrated around $\mu$, which indicates small phase errors, and setting $\mu=0$ implies that the phase error is unbiased. Although in theory $\kappa \in[0, \infty)$, typical values of $\kappa$ occupy a smaller bounded range. For example, least square channel estimation (LSCE) using a single pilot symbol provides $\kappa=[1.25,3,8,25,250]$ for $\mathrm{SNR}=[-5,0,5,10,20]$, respectively.

In the following subsections, the exact PDF $f_{B_{L}}\left(b_{L}\right)$ is derived for the cases of $L=2,3$, and the PDFs for $L \geq 4$ are approximated using the Central Limit Theorem (CLT). Moreover, for all scenarios, the phase error will be considered unbiased, $\mu_{i}=0 \forall i$, and the values of $\kappa_{i}$ are considered unequal deterministic variables. The phase errors $\epsilon_{i} \forall i$ are i.i.d. von Mises random variables. 
A. The Signal Envelope PDF for $L=2, f_{B_{2}}\left(b_{2}\right)$

For $L=2, \zeta_{L-1}=\zeta_{1}=\epsilon_{1}$ and $b_{1}=A_{1}$. Substituting these terms in (12) and rearranging the order of integration gives

$$
f_{B_{2}}\left(b_{2}\right)=\frac{b_{2}}{4 \pi^{3} I_{0}^{(2)}(\boldsymbol{\kappa})} \int_{-\infty}^{\infty} \mathrm{e}^{-j t\left(b_{2}^{2}-A_{1}^{2}-A_{2}^{2}\right)} \int_{-\pi}^{\pi} \mathrm{e}^{\kappa_{1} \cos \left(\epsilon_{1}\right)}\left[\int_{-\pi}^{\pi} \mathrm{e}^{2 j t A_{1} A_{2} \cos \left(\epsilon_{2}-\epsilon_{1}\right)+\kappa_{2} \cos \left(\epsilon_{2}\right)} d \epsilon_{2}\right] d \epsilon_{1} d t
$$

It should be noted that when the phases distribution is not uniform, it can't be assumed that one of theses phases is zero as reported in [48], [49], which implies that there is an additional integration that should be solved for the von Mises PDF. The integral inside the brackets can be evaluated with respect to $\epsilon_{2}$ as $[62,3.338 .4]$

$$
\begin{aligned}
\mathcal{I}_{2} & =\int_{-\pi}^{\pi} \mathrm{e}^{2 j t A_{1} A_{2} \cos \left(\epsilon_{2}-\epsilon_{1}\right)+\kappa_{2} \cos \left(\epsilon_{2}\right)} d \epsilon_{2} \\
& =2 \pi I_{0}\left(2\left|A_{1} A_{2}\right| \sqrt{-t^{2}+K^{2}+2 j t K \cos \left(\epsilon_{1}\right)}\right) .
\end{aligned}
$$

By substituting (15) in (14) and rearranging the integration order we obtain

$$
f_{B_{2}}\left(b_{2}\right)=\frac{b_{2}}{4 \pi^{3} I_{0}^{(2)}(\boldsymbol{\kappa})} \int_{-\pi}^{\pi} \mathrm{e}^{\kappa_{1} \cos \left(\epsilon_{1}\right)}\left[\int_{-\infty}^{\infty} \mathrm{e}^{-j t \tilde{\alpha}} \mathcal{I}_{2} d t\right] d \epsilon_{1}
$$

The integral inside the brackets, $\mathcal{I}_{3}$, can be evaluated using the following tabulated integral $[62,6.616 .1]$

$$
\int_{0}^{\infty} \mathrm{e}^{-\alpha x} J_{0}\left(\beta \sqrt{x^{2}+2 \lambda x}\right) d x=\frac{1}{\sqrt{\alpha^{2}+\beta^{2}}} \mathrm{e}^{\lambda\left(\alpha-\sqrt{\alpha^{2}+\beta^{2}}\right)}
$$

which after some straightforward manipulations can be written as,

$$
\int_{-\infty}^{\infty} \mathrm{e}^{-\alpha x} I_{0}\left(-j \beta \sqrt{x^{2}+2 \lambda x}\right) d x=\frac{1}{\sqrt{\alpha^{2}+\beta^{2}}}\left[\mathrm{e}^{\lambda\left(\alpha-\sqrt{\alpha^{2}+\beta^{2}}\right)}+\mathrm{e}^{\lambda\left(\alpha+\sqrt{\alpha^{2}+\beta^{2}}\right)}\right] .
$$

By using the change of variable $x=t+v$,

$$
\int_{-\infty}^{\infty} \mathrm{e}^{-\alpha x} I_{0}\left(-j \beta \sqrt{x^{2}+2 \lambda x}\right) d x=\mathrm{e}^{-\alpha v} \int_{-\infty}^{\infty} \mathrm{e}^{-\alpha t} I_{0}\left(-j \beta \sqrt{t^{2}+2 t(v+\lambda)+v^{2}+2 \lambda v}\right) d t .
$$

Therefore, $\mathcal{I}_{3}$ can be evaluated as

$$
\mathcal{I}_{3}=\mathrm{e}^{\alpha v} \frac{2 \pi}{\mathcal{B}}\left[\mathrm{e}^{\lambda(\alpha-\mathcal{B})}+\mathrm{e}^{\lambda(\alpha+\mathcal{B})}\right], \quad \alpha^{2}+\beta^{2} \geq 0 .
$$


By noting that $v+\lambda=-j K \cos \left(\epsilon_{1}\right)$ and $v^{2}+2 \lambda v=-K^{2}, f_{B_{2}}\left(b_{2}\right)$ can be expressed as

$$
\begin{aligned}
f_{B_{2}}\left(b_{2}\right) & =\frac{b_{2}}{2 \pi^{2} \mathcal{B} I_{0}^{(2)}(\boldsymbol{\kappa})} \int_{-\pi}^{\pi}\left[\mathrm{e}^{\left[\left(K \tilde{\alpha}+\kappa_{1}\right) \cos \left(\epsilon_{1}\right)-K \mathcal{B} \sin \left(\epsilon_{1}\right)\right]}+\int_{-\pi}^{\pi} \mathrm{e}^{\left[\left(K \tilde{\alpha}+\kappa_{1}\right) \cos \left(\epsilon_{1}\right)+K \mathcal{B} \sin \left(\epsilon_{1}\right)\right]}\right] d \epsilon_{1} \\
& =\frac{b_{2}}{\pi \mathcal{B} I_{0}^{(2)}(\boldsymbol{\kappa})}\left[I_{0}\left[\sqrt{\left(K \tilde{\alpha}+\kappa_{1}\right)^{2}+[K \mathcal{B}]^{2}}\right]+I_{0}\left[\sqrt{\left(K \tilde{\alpha}+\kappa_{1}\right)^{2}+[K \mathcal{B}]^{2}}\right]\right] \\
& =\frac{2 b_{2}}{\pi \mathcal{B} I_{0}^{(2)}(\boldsymbol{\kappa})} I_{0}\left[\sqrt{\left(2 K A_{1} A_{2} \mathcal{B}_{2}+\kappa_{1}\right)^{2}+K^{2}\left(-4 A_{1}^{2} A_{2}^{2} \mathcal{B}_{2}^{2}+4 A_{1}^{2} A_{2}^{2}\right)}\right] \\
& =\frac{2 b_{2}}{\pi \mathcal{B} I_{0}^{(2)}(\boldsymbol{\kappa})} I_{0}\left[\sqrt{\left(\kappa_{1}-\kappa_{2}\right)^{2}+\frac{\kappa_{2} \kappa_{1}}{A_{1} A_{2}}\left(b_{2}^{2}-\mathcal{A}_{2}\right)}\right]
\end{aligned}
$$

which after some algebraic simplifications, it can be simplified to

$$
\begin{aligned}
f_{B_{2}}\left(b_{2}\right) & =\frac{2 b_{2}}{\pi \mathcal{B} I_{0}^{(2)}(\boldsymbol{\kappa})} I_{0}\left[\sqrt{\kappa_{1}^{2}+\kappa_{2}^{2}+2 \kappa_{2} \kappa_{1} \mathcal{B}_{2}}\right],\left|\mathcal{B}_{2}\right| \leq 1 \\
& =\frac{2 b_{2} I_{0}\left[\sqrt{\left(\kappa_{1}-\kappa_{2}\right)^{2}+\frac{\kappa_{2} \kappa_{1}}{A_{1} A_{2}}\left(b_{2}^{2}-\mathcal{A}_{2}\right)}\right]}{\pi \sqrt{\left(\mathcal{A}_{3}-b_{2}^{2}\right)\left(b_{2}^{2}-\mathcal{A}_{2}\right)} I_{0}^{(2)}(\boldsymbol{\kappa})},\left|\mathcal{B}_{2}\right| \leq 1 .
\end{aligned}
$$

The condition $\left|\mathcal{B}_{2}\right| \leq 1$ can be solved as

$$
\left|\frac{b_{2}^{2}-A_{1}^{2}-A_{2}^{2}}{2 A_{1} A_{2}}\right| \leq 1
$$

which gives

$$
B_{2, \mathrm{~m}} \leq b_{2} \leq B_{2, \mathrm{M}}
$$

where $B_{2, \mathrm{~m}}=\left|A_{1}-A_{2}\right|$ and $B_{2, \mathrm{M}}=A_{1}+A_{2}$. It can be noted that $f_{B_{2}}\left(b_{2}\right)$ derived in [48], [49] is just a special case of (22) where $\kappa_{i}=0 \forall i$.

Fig. 2a shows the PDFs of $f_{B_{2}}\left(b_{2}\right)$ where the individual signal amplitudes $A_{i}=1 \forall i$. As can be noted from the figure, the derived formula in (22) matches the simulation results for all the considered values of $\kappa$, including $\kappa=0$, which corresponds to the uniformly distributed phases. The figure shows that large phase errors may drive the amplitude $B_{2}$ below the $\min \left\{A_{1}, A_{2}\right\}$, which implies that the error rate would be worse than the case without IRS. Increasing the value of $\kappa$, makes the envelope more concentrated around $A_{1}+A_{2}$, which implies that the IRS will provide a gain of about $6 \mathrm{~dB}$ in terms of SNR. 


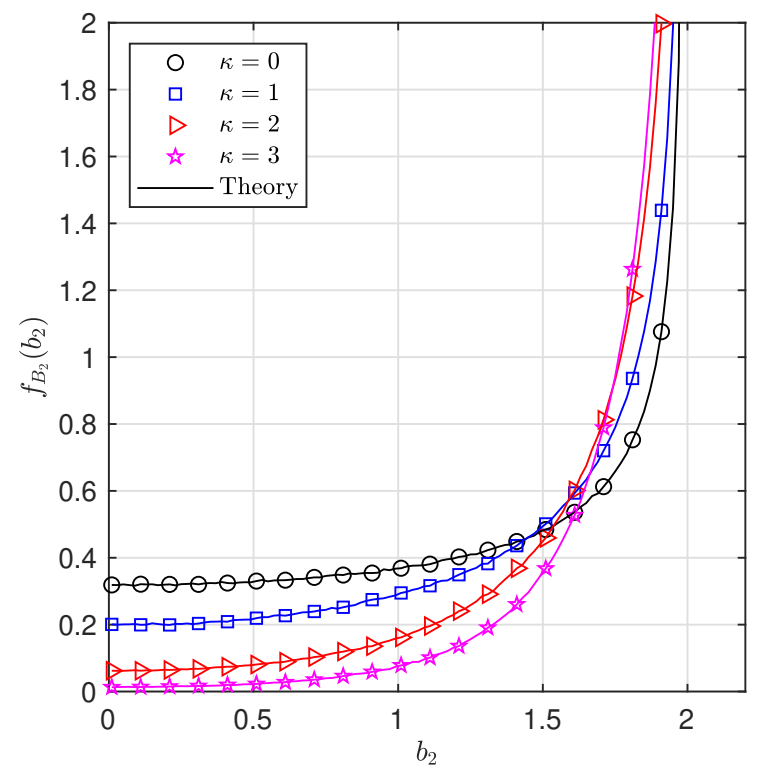

(a) $L=2$

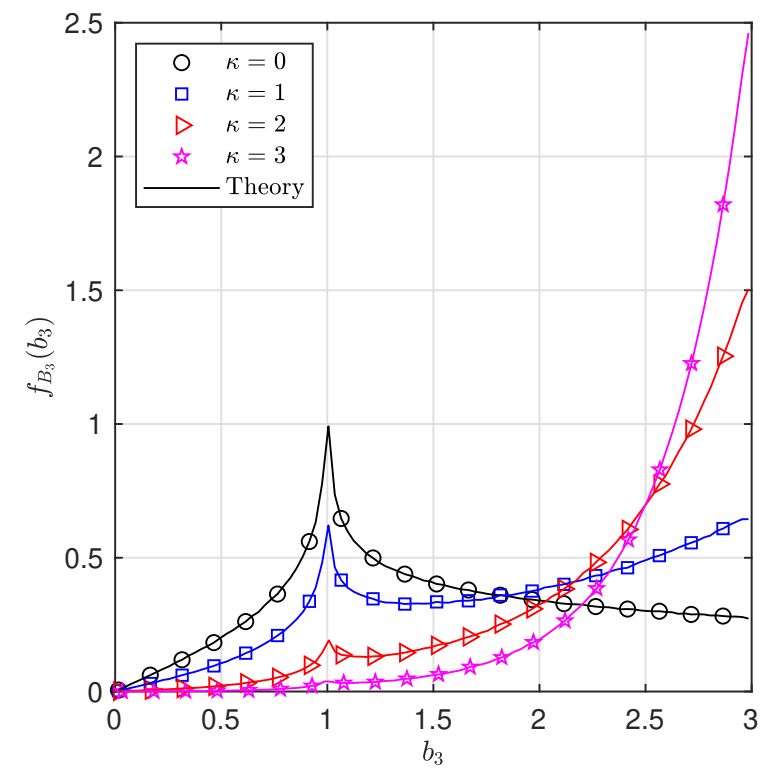

(b) $L=3$

Fig. 2: The PDF of the signal envelope for different values of $\kappa$ for $L=2,3, A_{i}=1 \forall i$.

\section{B. The Signal Envelope PDF for $L=3, f_{B_{3}}\left(b_{3}\right)$}

Using the same assumptions of $L=2, f_{B_{3}}\left(b_{3}\right)$ can be expressed as

$$
f_{B_{3}}\left(b_{3}\right)=\frac{b_{3}}{8 \pi^{4} I_{0}^{(3)}(\boldsymbol{\kappa})} \int_{-\infty}^{\infty} \mathrm{e}^{-j b_{3}^{2} t} \int_{-\pi}^{\pi} \int_{-\pi}^{\pi} \int_{-\pi}^{\pi} \mathrm{e}^{j t\left\{b_{2}^{2}+A_{3}^{2}+2 A_{3} b_{2} \cos \left(\epsilon_{3}-\zeta_{2}\right)\right\}} \mathrm{e}^{\sum_{i=1}^{L} \kappa_{i} \cos \left(\epsilon_{i}\right)} d \epsilon_{1} d \epsilon_{2} d \epsilon_{3} d t .
$$

where $b_{2}^{2}=A_{1}^{2}+A_{2}^{2}+2 A_{1} A_{2} \cos \left(\epsilon_{2}-\epsilon_{1}\right)$ and $\zeta_{2}=\tan ^{-1}\left[\frac{A_{1} \sin \left(\epsilon_{1}\right)+A_{2} \sin \left(\epsilon_{2}\right)}{A_{1} \cos \left(\epsilon_{1}\right)+A_{2} \cos \left(\epsilon_{2}\right)}\right]$. Hence, the constraint $\left|\mathcal{B}_{2}\right| \leq 1$ is also applicable. By substituting the identity

$$
\cos \left(\epsilon_{3}-\zeta_{2}\right)=\cos \left(\epsilon_{3}\right) \cos \left(\zeta_{2}\right)+\sin \left(\epsilon_{3}\right) \sin \left(\zeta_{2}\right)
$$

and evaluating the integral with respect to $\epsilon_{3}$ gives

$$
\begin{aligned}
f_{B_{3}}\left(b_{3}\right)=\frac{b_{3}}{4 \pi^{3} I_{0}^{(3)}(\boldsymbol{\kappa})} & \int_{-\infty}^{\infty} \mathrm{e}^{-j t\left(b_{3}^{2}-A_{3}^{2}\right)} \int_{-\pi}^{\pi} \int_{-\pi}^{\pi} \mathrm{e}^{j t b_{2}^{2}} \mathrm{e}^{\kappa_{1} \cos \left(\epsilon_{1}\right)+\kappa_{2} \cos \left(\epsilon_{2}\right)} \\
& \times I_{0}\left[\sqrt{\left[2 j t A_{3} b_{2} \cos \left(\zeta_{2}\right)+\kappa_{3}\right]^{2}+\left[2 j t A_{3} b_{2} \sin \left(\zeta_{2}\right)\right]^{2}}\right] d \epsilon_{1} d \epsilon_{2} d t \\
=\frac{b_{3}}{4 \pi^{3} I_{0}^{(3)}(\boldsymbol{\kappa})} & \int_{-\pi}^{\pi} \mathrm{e}^{\kappa_{1} \cos \left(\epsilon_{1}\right)} \int_{-\pi}^{\pi} \frac{1}{\overline{\mathcal{B}}} \mathrm{e}^{\kappa_{2} \cos \left(\epsilon_{2}\right)} \\
& \times\left[\mathrm{e}^{-\dot{K} \sin \left(\zeta_{2}\right) \dot{\mathcal{B}}+\check{\alpha} \dot{K} \cos \left(\zeta_{2}\right)}+\mathrm{e}^{\dot{K} \sin \left(\zeta_{2}\right) \dot{\mathcal{B}}+\check{\alpha} \dot{K} \cos \left(\zeta_{2}\right)}\right] \mathbf{1}_{\left\{\left|\mathcal{B}_{3}\right| \leq 1\right\}} d \epsilon_{2} d \epsilon_{1}
\end{aligned}
$$


$\mathbf{1}_{\{\cdot\}}$ is the indicator function of the set $\{\cdot\}$. Thus,

$$
f_{B_{3}}\left(b_{3}\right)=\frac{b_{3}}{2 \pi^{3} I_{0}^{(3)}(\boldsymbol{\kappa})} \int_{-\pi}^{\pi} \int_{-\pi}^{\pi} \frac{1}{\overline{\mathcal{B}}^{\prime}} \mathrm{e}^{\kappa_{1} \cos \left(\epsilon_{1}\right)+\kappa_{2} \cos \left(\epsilon_{2}\right)}\left[\mathrm{e}^{\dot{K} \check{\alpha} \cos \left(\zeta_{2}\right)} \cosh \left(\dot{K} \sin \left(\zeta_{2}\right) \dot{\mathcal{B}}\right)\right] \mathbf{1}_{\left\{\left|\mathcal{B}_{3}\right| \leq 1\right\}} d \epsilon_{1} d \epsilon_{2} .
$$

As can be noted from (27), deriving $f_{B_{3}}\left(b_{3}\right)$ in closed-form is intractable. Fig. 2 b shows $f_{B_{3}}\left(b_{3}\right)$ for various values of $\kappa$, where high values of $\kappa$ leads the PDF to be mostly concentrated close to the maximum amplitude, i.e., $B_{3, \mathrm{M}}=A_{1}+A_{2}+A_{3}$. For the perfect phase estimations case, the anticipated gain provided by the IRS will be about $9.5 \mathrm{~dB}$. Nevertheless, for low values of $\kappa$, the signal will experience fading, which may cause severe increase to the probability of error. In such scenarios, the error probability without IRS may become less than that with IRS.

\section{The Signal Envelope PDF $f_{B_{L}}\left(b_{L}\right)$ for $L \geq 4$}

For large $L$ values, the CLT can be invoked to compute the PDF $f_{B_{L}}\left(b_{L}\right)$. However, by referring to (7), it is easier to compute $f_{B_{L}^{2}}\left(b_{L}^{2}\right)$. Moreover, because the values of $B_{L}$ are bounded by $B_{L, \mathrm{~m}} \leq b_{L} \leq$ $B_{L, \mathrm{M}}$, it will be more accurate to use the truncated Gaussian distribution [63] to derive the PDF using CLT. By defining $y_{L} \triangleq b_{L}^{2}$ for notational simplicity, we obtain

$$
f_{Y_{L}}\left(y_{L}\right)=\frac{1}{\varpi_{L} \sqrt{2 \pi \sigma_{Y_{L}}^{2}}} \exp \left(-\frac{\left(y_{L}-m_{Y_{L}}\right)^{2}}{2 \sigma_{Y_{L}}^{2}}\right)
$$

where $\varpi_{L}$ is the truncated PDF normalization factor, which is approximately unity for high values of $m_{Y_{L}}\left[63\right.$, p. 20], $m_{Y_{L}}$ and $\sigma_{Y_{L}}^{2}$ are the mean and variance of the Gaussian PDF, which are given by

$$
m_{Y_{L}}=\sum_{i=1}^{L} A_{i}^{2}+2 \sum_{L \geq j>k \geq 1} A_{j} A_{k} \frac{I_{1}\left(\kappa_{j}\right) I_{1}\left(\kappa_{k}\right)}{I_{0}\left(\kappa_{j}\right) I_{0}\left(\kappa_{k}\right)}
$$

and

$$
\sigma_{Y_{L}}^{2} \triangleq \mathrm{E}\left[Y_{L}^{2}\right]-m_{Y_{L}}^{2}
$$

where

$$
\begin{array}{r}
\mathrm{E}\left[Y_{L}^{2}\right]=\|\mathbf{A}\|^{4}+4 \sum_{L \geq j>k \geq 1} \frac{A_{j}^{2} A_{k}^{2}}{2}\left(1+\frac{I_{2}\left(\kappa_{j}\right)}{I_{0}\left(\kappa_{j}\right)} \frac{I_{2}\left(\kappa_{k}\right)}{I_{0}\left(\kappa_{k}\right)}\right)+4\|\mathbf{A}\|^{2} \sum_{n \geq j>k \geq 1} A_{j} A_{k} \frac{I_{1}\left(\kappa_{j}\right) I_{1}\left(\kappa_{k}\right)}{I_{0}\left(\kappa_{j}\right) I_{0}\left(\kappa_{k}\right)} \\
\quad+4 \sum_{L \geq j>k \geq 1} \sum_{L \geq i>l>1} A_{j} A_{k} A_{i} A_{l} \frac{I_{1}\left(\kappa_{j}\right) I_{1}\left(\kappa_{k}\right) I_{1}\left(\kappa_{i}\right) I_{1}\left(\kappa_{l}\right)}{I_{0}\left(\kappa_{j}\right) I_{0}\left(\kappa_{k}\right) I_{0}\left(\kappa_{i}\right) I_{0}\left(\kappa_{l}\right)} .
\end{array}
$$

The derivation of $m_{Y_{L}}$ and $\sigma_{Y_{L}}^{2}$ is given in Appendix II. 


\section{SER ANALYSIS}

A. One Reflector, $L=1$

In this case, $b_{1}=\beta_{1} h_{1} \hbar_{1} \triangleq A_{1}$, hence the channel is deterministic and based on (10), the instantaneous SNR is $\gamma_{1}=\frac{A_{1}^{2}}{\sigma_{z}^{2}}$. Therefore, the SER can be expressed as

$$
\bar{P}_{\mathrm{e}}=C_{1} Q\left(\sqrt{C_{2} \gamma_{1}}\right)
$$

where $Q(\cdot)$ is the tail distribution function of the standard normal distribution, $C_{1}$ and $C_{2}$ are constants that depend on the modulation scheme [64, Table 6.1, pp. 179].

B. Two Reflectors, $L=2$

Because the phase between the two reflectors is random, then the instantaneous SNR $\gamma_{2}=\frac{b_{2}^{2}}{\sigma_{z}^{2}}$ is also random. Therefore, the average SER $\bar{P}_{\mathrm{e}}$ can be expressed as

$$
\begin{aligned}
\bar{P}_{\mathrm{e}} & =C_{1} \int_{B_{2, \mathrm{~m}}}^{B_{2, \mathrm{M}}} Q\left(b_{2} \sqrt{\frac{C_{2}}{\sigma_{z}^{2}}}\right) f_{B_{2}}\left(b_{2}\right) d b_{2} \\
& =\frac{2 C_{1}}{\pi I_{0}^{(2)}(\boldsymbol{\kappa})} \int_{B_{2, \mathrm{~m}}}^{B_{2, \mathrm{M}}} \frac{b_{2} Q\left(b_{2} \sqrt{\frac{C_{2}}{\sigma_{z}^{2}}}\right)}{\sqrt{\left(\mathcal{A}_{3}-b_{2}^{2}\right)\left(b_{2}^{2}-\mathcal{A}_{2}\right)}} I_{0}\left[\sqrt{\left(\kappa_{1}-\kappa_{2}\right)^{2}+\frac{\kappa_{2} \kappa_{1}}{A_{1} A_{2}}\left(b_{2}^{2}-\mathcal{A}_{2}\right)}\right] d b_{2} .
\end{aligned}
$$

By substituting $f_{B_{2}}\left(b_{2}\right)(22)$ into (33), substituting $x=\sqrt{b_{2}^{2}-\mathcal{A}_{2}}$, and noting that $B_{2, \mathrm{~m}}=\left|A_{1}-A_{2}\right|$ and $B_{2, \mathrm{M}}=\left(A_{1}+A_{2}\right)$, then $\bar{P}_{\mathrm{e}}$ can be written as

$$
\bar{P}_{\mathrm{e}}=\frac{2 C_{1}}{\pi I_{0}^{(2)}(\boldsymbol{\kappa})} \int_{0}^{\mathcal{A}_{1}} \frac{Q\left(\sqrt{\frac{C_{2}}{\sigma_{z}^{2}}\left(x^{2}+\mathcal{A}_{2}\right)}\right)}{\sqrt{-x^{2}+\mathcal{A}_{1}^{2}}} I_{0}\left[\sqrt{\left(\kappa_{1}-\kappa_{2}\right)^{2}+\frac{\kappa_{2} \kappa_{1}}{A_{1} A_{2}} x^{2}}\right] d x .
$$

Evaluating the integral in (34) is intractable due to the Bessel function. Therefore, we use the infinite series representation of $I_{0}(\cdot)$, which gives

$$
\bar{P}_{\mathrm{e}}=\frac{2 C_{1}}{\pi I_{0}^{(2)}(\boldsymbol{\kappa})} \sum_{m=0}^{\infty} \frac{1}{2^{2 m}(m !)^{2}} \int_{0}^{\mathcal{A}_{1}} \frac{Q\left(\sqrt{\frac{C_{2}}{\sigma_{z}^{2}}\left(x^{2}+\mathcal{A}_{2}\right)}\right)}{\sqrt{-x^{2}+\mathcal{A}_{1}^{2}}}\left[\left(\kappa_{1}-\kappa_{2}\right)^{2}+\frac{\kappa_{2} \kappa_{1}}{A_{1} A_{2}} x^{2}\right]^{m} d x .
$$

Using the binomial theorem (35) can be expressed as

$$
\bar{P}_{\mathrm{e}}=\frac{2 C_{1}}{\pi I_{0}^{(2)}(\boldsymbol{\kappa})} \sum_{m=0}^{\infty} \frac{1}{2^{2 m}(m !)^{2}} \sum_{k=0}^{m}\left(\begin{array}{c}
m \\
k
\end{array}\right)\left(\kappa_{1}-\kappa_{2}\right)^{2(m-k)}\left(\frac{\kappa_{2} \kappa_{1}}{A_{1} A_{2}}\right)^{k} \int_{0}^{\mathcal{A}_{1}} \frac{Q\left(\sqrt{\frac{C_{2}}{\sigma_{z}^{2}}\left(x^{2}+\mathcal{A}_{2}\right)}\right)}{\sqrt{-x^{2}+\mathcal{A}_{1}^{2}}} x^{2 k} d x
$$


However, the integral in (36) does not have a closed-form solution except for the special case where $A_{1}=A_{2} \triangleq A$. Consequently, $\bar{P}_{\mathrm{e}}$ is reduced to

$$
\bar{P}_{\mathrm{e}}=\frac{2 C_{1}}{\pi I_{0}^{(2)}(\boldsymbol{\kappa})} \sum_{m=0}^{\infty} \frac{1}{2^{2 m}(m !)^{2}} \sum_{k=0}^{m}\left(\begin{array}{c}
m \\
k
\end{array}\right)\left(\kappa_{1}-\kappa_{2}\right)^{2(m-k)}\left(\frac{\kappa_{2} \kappa_{1}}{A^{2}}\right)^{k} \int_{0}^{\mathcal{A}_{1}} \frac{Q\left(\sqrt{\frac{C_{2}}{\sigma_{z}^{2}}} x\right)}{\sqrt{-x^{2}+\mathcal{A}_{1}^{2}}} x^{2 k} d x
$$

which can be evaluated as [65, 2.8.3.1, pp. 102],

$$
\begin{aligned}
& \bar{P}_{\mathrm{e}}=\frac{C_{1}}{\pi I_{0}^{(2)}(\boldsymbol{\kappa})} \sum_{m=0}^{\infty} \frac{1}{2^{2 m}(m !)^{2}} \sum_{k=0}^{m}\left(\begin{array}{c}
m \\
k
\end{array}\right)\left(\kappa_{1}-\kappa_{2}\right)^{2(m-k)}\left(\frac{\kappa_{2} \kappa_{1}}{A^{2}}\right)^{k} \\
& \times\left\{\frac{\mathcal{A}_{1}^{2 k}}{2} \mathrm{~B}\left(k+\frac{1}{2}, \frac{1}{2}\right)-\sqrt{\frac{C_{2}}{2 \pi \sigma_{z}^{2}}} \mathcal{A}_{1}^{2 k+1} \mathrm{~B}\left(k+1, \frac{1}{2}\right){ }_{2} F_{2}\left(\left[k+1, \frac{1}{2}\right] ;\left[k+\frac{3}{2}, \frac{3}{2}\right] ;-\frac{\mathcal{A}_{1}^{2} C_{2}}{2 \sigma_{z}^{2}}\right)\right\}
\end{aligned}
$$

where ${ }_{2} F_{2}(\cdot)$ is the hypergeometric function and $\mathrm{B}(\cdot, \cdot)$ is the beta function, $\mathrm{B}(\alpha, \beta)=\frac{\Gamma(\alpha) \Gamma(\beta)}{\Gamma(\alpha+\beta)}$.

For the general case of $A_{1} \neq A_{2}$, the integral can be solved using the $Q$-function approximation [66]

$$
Q(x) \simeq \sum_{l=1}^{N} \delta_{l} \exp \left(-\varepsilon_{l} x^{2}\right), x>0
$$

where $\delta_{l}$ and $\varepsilon_{l}$ are constants evaluated to minimize the approximation error, their values are given in [66]. Using the $Q$-function approximation (36) can be simplified to

$$
\begin{array}{r}
\bar{P}_{\mathrm{e}}=\frac{2 C_{1}}{\pi I_{0}^{(2)}(\boldsymbol{\kappa})} \sum_{m=0}^{\infty} \frac{1}{2^{2 m}(m !)^{2}} \sum_{k=0}^{m}\left(\begin{array}{c}
m \\
k
\end{array}\right)\left(\kappa_{1}-\kappa_{2}\right)^{2(m-k)}\left(\frac{\kappa_{2} \kappa_{1}}{A_{1} A_{2}}\right)^{k} \sum_{l=1}^{N} \delta_{l} \exp \left(-\frac{C_{2} \mathcal{A}_{2}}{\sigma_{z}^{2}} \varepsilon_{l}\right) \\
\times \int_{0}^{\mathcal{A}_{1}} \frac{\exp \left(-\frac{\varepsilon_{l} C_{2}}{\sigma_{z}^{2}} x^{2}\right)}{\sqrt{-x^{2}+\mathcal{A}_{1}^{2}}} x^{2 k} d x
\end{array}
$$

Substituting $u=x^{2}$ yields

$$
\begin{array}{r}
\bar{P}_{\mathrm{e}}=\frac{C_{1}}{\pi I_{0}^{(2)}(\boldsymbol{\kappa})} \sum_{m=0}^{\infty} \frac{1}{2^{2 m}(m !)^{2}} \sum_{k=0}^{m}\left(\begin{array}{c}
m \\
k
\end{array}\right)\left(\kappa_{1}-\kappa_{2}\right)^{2(m-k)}\left(\frac{\kappa_{2} \kappa_{1}}{A_{1} A_{2}}\right)^{k} \sum_{l=1}^{N} \delta_{l} \exp \left(-\frac{C_{2} \mathcal{A}_{2}}{\sigma_{z}^{2}} \varepsilon_{l}\right) \\
\times \int_{0}^{\mathcal{A}_{1}^{2}} \frac{\exp \left(-\frac{\varepsilon_{l} C_{2}}{\sigma_{z}^{2}} u\right)}{\sqrt{-u+\mathcal{A}_{1}^{2}}} u^{k-0.5} d u
\end{array}
$$

Thereafter, [67, 2.3.6.1, pp. 324] is used to solve the integral, which gives,

$$
\begin{array}{r}
\bar{P}_{\mathrm{e}}=\frac{C_{1}}{\pi I_{0}^{(2)}(\boldsymbol{\kappa})} \sum_{m=0}^{\infty} \frac{1}{2^{2 m}(m !)^{2}} \sum_{k=0}^{m}\left(\begin{array}{c}
m \\
k
\end{array}\right)\left(\kappa_{1}-\kappa_{2}\right)^{2(m-k)}\left(\frac{\kappa_{2} \kappa_{1}}{A_{1} A_{2}}\right)^{k} \sum_{l=1}^{N} \delta_{l} \exp \left(-\frac{C_{2} \mathcal{A}_{2}}{\sigma_{z}^{2}} \varepsilon_{l}\right) \\
\times \mathrm{B}(k+0.5,0.5)\left(4 A_{1} A_{2}\right)^{k}{ }_{1} F_{1}\left(k+0.5, k+1,-\mathcal{A}_{1}^{2} \frac{\varepsilon_{l} C_{2}}{\sigma_{z}^{2}}\right) .
\end{array}
$$


C. Three Reflectors, $L=3$

Similar to the $L=2$ case, the SER for $L=3$ can be computed as,

$$
\bar{P}_{\mathrm{e}}=C_{1} \int_{B_{3, \mathrm{~m}}}^{B_{3, \mathrm{M}}} Q\left(b_{3} \sqrt{\frac{C_{2}}{\sigma_{z}^{2}}}\right) f_{B_{3}}\left(b_{3}\right) d b_{3}
$$

where $B_{3, \mathrm{~m}}$ and $B_{3, \mathrm{M}}$ the minimum and maximum values of $B_{3}$. As can be noted from (27), evaluating $\bar{P}_{\mathrm{e}}$ in closed-form is infeasible because $f_{B_{3}}\left(b_{3}\right)$ does not have a closed-form solution. Consequently, the SER can be obtained numerically after substituting (27) into (43).

D. Number of Reflectors $L \geq 4$

Because $B_{L, \mathrm{~m}} \leq B_{L} \leq B_{L, \mathrm{M}}$, then $Y_{L}=B_{L}^{2}$ is bounded as $B_{L, \mathrm{~m}}^{2} \leq Y_{L} \leq B_{L, \mathrm{M}}^{2}$. Therefore,

$$
\begin{aligned}
\bar{P}_{\mathrm{e}} & =\int_{B_{L, \mathrm{~m}}^{2}}^{B_{L, \mathrm{M}}^{2}} P_{\mathrm{e}} f_{Y_{L}}\left(y_{L}\right) d y_{L} \\
& =\frac{C_{1}}{\sqrt{2 \pi \sigma_{Y_{L}}^{2}}} \int_{B_{L, \mathrm{~m}}^{2}}^{B_{L, \mathrm{M}}^{2}} Q\left(\sqrt{\frac{C_{2}}{\sigma_{z}^{2}} y_{L}}\right) \exp \left(-\frac{\left(y_{L}-\mu_{Y_{L}}\right)^{2}}{2 \sigma_{Y_{L}}^{2}}\right) d y_{L} .
\end{aligned}
$$

To be able to solve the integral, the $Q$-function approximation is used [68]. and thus

$$
\begin{aligned}
\bar{P}_{\mathrm{e}}=\frac{C_{1} \exp \left(-\frac{\mu_{Y_{L}}^{2}}{2 \sigma_{Y_{L}}^{2}}\right)}{1.135 \pi \sqrt{2 \sigma_{Y_{L}}^{2}}} \sum_{i=1}^{n_{a}} \frac{(-1)^{i+1} 1.98^{i}}{i ! 2^{\frac{i+1}{2}}}\left(\frac{C_{2}}{\sigma_{z}^{2}}\right)^{\frac{i-1}{2}} \\
\quad \times \int_{B_{L, \mathrm{~m}}^{2}}^{B_{L, \mathrm{M}}^{2}} y_{L}^{\frac{i-1}{2}} \exp \left(-\frac{y_{L}^{2}}{2 \sigma_{Y_{L}}^{2}}+\left(\frac{\mu_{Y_{L}}}{\sigma_{Y_{L}}^{2}}-\frac{C_{2}}{2 \sigma_{z}^{2}}\right) y_{L}\right) d y_{L}
\end{aligned}
$$

The integral in (45) can be solved recursively [67, 1.3.3.19, pp. 140], and the solution is given in terms of the error function or $Q$-function. However, when $B_{L, \mathrm{~m}}^{2} \rightarrow 0$ and $B_{L, \mathrm{M}}^{2} \rightarrow \infty, \bar{P}_{\mathrm{e}}$ can be given as [67, 2.3.15.3, pp. 343]

$$
\bar{P}_{\mathrm{e}}=Z \sum_{i=1}^{n_{a}} \frac{(-1)^{i+1}}{1.98^{-i} i !} \Gamma\left(\frac{i+1}{2}\right)\left(\frac{\sigma_{Y_{L}} C_{2}}{2 \sigma_{z}^{2}}\right)^{\frac{i}{2}} D_{-\left(\frac{i+1}{2}\right)}\left(\sigma_{Y_{L}}\left(\frac{C_{2}}{2 \sigma_{z}^{2}}-\frac{\mu_{Y_{L}}}{\sigma_{Y_{L}}^{2}}\right)\right)
$$

where

$$
Z=\frac{C_{1} \sigma_{z}}{2 \times 1.135 \pi \sqrt{C_{2} \sigma_{Y_{L}}}} \exp \left(\frac{\sigma_{Y_{L}}^{2}}{4}\left(\frac{\mu_{Y_{L}}}{\sigma_{Y_{L}}^{2}}-\frac{C_{2}}{2 \sigma_{z}^{2}}\right)^{2}-\frac{\mu_{Y_{L}}^{2}}{2 \sigma_{Y_{L}}^{2}}\right)
$$

and $D_{(\cdot)}(\cdot)$ is the parabolic cylinder function. 


\section{Outage Probability Analysis}

For the case of $L=1$, the channel gain is fixed, and thus, the outage process depends only on the signal power. For the remaining cases, i.e., $L=2, L=3$ and $L \geq 4$, the derivation of the outage probability is presented below.

\section{A. Two Reflectors, $L=2$}

Given that the instantaneous SNR threshold $\gamma_{O} \triangleq b_{O}^{2} / \sigma_{z}^{2}$, then the envelope threshold $b_{O}=\sqrt{\sigma_{z}^{2} \gamma_{O}}$. Therefore, the outage probability can be derived as

$$
\bar{P}_{O}= \begin{cases}\int_{B_{2, \mathrm{~m}}}^{b_{O}} f_{B_{2}}\left(b_{2}\right) d b_{2}, & b_{O}>B_{2, \mathrm{~m}} \\ 1, & b_{O} \leq B_{2, \mathrm{~m}}\end{cases}
$$

where $\left.\bar{P}_{O}\right|_{b_{O}>B_{2, \mathrm{~m}}}$ can be computed as

$$
\bar{P}_{O}=\frac{2}{\pi I_{0}^{(2)}(\boldsymbol{\kappa})} \int_{B_{2, \mathrm{~m}}}^{b_{O}} \frac{b_{2} I_{0}\left[\sqrt{\left(\kappa_{1}-\kappa_{2}\right)^{2}+\frac{\kappa_{2} \kappa_{1}}{A_{1} A_{2}}\left(b_{2}^{2}-\mathcal{A}_{2}\right)}\right]}{\sqrt{\left(\mathcal{A}_{3}-b_{2}^{2}\right)\left(b_{2}^{2}-\mathcal{A}_{2}\right)}} d b_{2}
$$

substituting $x=\sqrt{b_{2}^{2}-\mathcal{A}_{2}}$ and noting that $B_{2, \mathrm{~m}}^{2}=\mathcal{A}_{2}$ gives $d x=b / x d b$

$$
\bar{P}_{O}=\frac{2}{\pi I_{0}^{(2)}(\boldsymbol{\kappa})} \int_{0}^{\sqrt{b_{O}^{2}-\mathcal{A}_{2}}} \frac{I_{0}\left[\sqrt{\left(\kappa_{1}-\kappa_{2}\right)^{2}+\frac{\kappa_{2} \kappa_{1}}{A_{1} A_{2}} x^{2}}\right]}{\sqrt{-x^{2}+\mathcal{A}_{1}^{2}}} d x
$$

Using the infinite series representation of the Bessel function gives

$$
\bar{P}_{O}=\frac{2}{\pi I_{0}^{(2)}(\boldsymbol{\kappa})} \sum_{m=0}^{\infty} \frac{1}{2^{2 m}(m !)^{2}} \int_{0}^{\sqrt{b_{O}^{2}-\mathcal{A}_{2}}} \frac{\left(\left(\kappa_{1}-\kappa_{2}\right)^{2}+\frac{\kappa_{2} \kappa_{1}}{A_{1} A_{2}} x^{2}\right)^{m}}{\sqrt{-x^{2}+\mathcal{A}_{1}^{2}}} d x .
$$

Then, by applying the binomial series expansion,

$$
\bar{P}_{O}=\frac{2}{\pi I_{0}^{(2)}(\boldsymbol{\kappa})} \sum_{m=0}^{\infty} \frac{1}{2^{2 m}(m !)^{2}} \sum_{l=0}^{m}\left(\begin{array}{c}
m \\
l
\end{array}\right)\left(\kappa_{1}-\kappa_{2}\right)^{2(m-l)}\left(\frac{\kappa_{2} \kappa_{1}}{A_{1} A_{2}}\right)^{l} \int_{0}^{\sqrt{b_{O}^{2}-\mathcal{A}_{2}}} \frac{x^{2 l}}{\sqrt{-x^{2}+\mathcal{A}_{1}^{2}}} d x
$$

which can be solved using [67, 1.2.48.8, pp. 97] as

$$
\bar{P}_{O}=\frac{2}{\pi I_{0}^{(2)}(\boldsymbol{\kappa})} \sum_{m=0}^{\infty} \frac{1}{2^{2 m}(m !)^{2}}\left(\left.\bar{P}_{O}\right|_{l=0}+\left.\bar{P}_{O}\right|_{l \geq 1}\right)
$$


where $\left.\bar{P}_{O}\right|_{l=0}=\left(\kappa_{1}-\kappa_{2}\right)^{2 m} \arcsin \left(\frac{B_{O}}{\mathcal{A}_{1}}\right), B_{O}=\sqrt{b_{o}^{2}-\mathcal{A}_{2}}$, and

$$
\begin{array}{r}
\left.\bar{P}_{O}\right|_{l \geq 1}=\sum_{l=1}^{m}\left(\begin{array}{c}
m \\
l
\end{array}\right)\left(\kappa_{1}-\kappa_{2}\right)^{2(m-l)}\left(\frac{\kappa_{2} \kappa_{1}}{A_{1} A_{2}}\right)^{l}\left\{\left[B_{O}^{2 l-1}+\sum_{k=1}^{l-1} H_{k, l} \mathcal{A}_{1}^{2 k} B_{O}^{2 l-2 k-1}\right]\right. \\
\left.\times \frac{-1}{2 l} \sqrt{\mathcal{A}_{1}^{2}-B_{O}^{2}}+\frac{\mathcal{A}_{1}^{2 l}(2 l-1) ! !}{2^{l} l !} \arcsin \left(\frac{B_{O}}{\mathcal{A}_{1}}\right)\right\}
\end{array}
$$

where

$$
H_{k, l}=\frac{(2 l-1)(2 l-3) \cdots(2 l-2 k+1)}{2^{k}(l-1)(l-2) \cdots(l-k)}
$$

and $(\cdot) ! !$ is the double factorial.

B. Three Reflectors, $L=3$

The outage probability for this case can be derived as

$$
\bar{P}_{O}=\left\{\begin{array}{ll}
\int_{B_{3, \mathrm{~m}}}^{b_{O}} f_{B_{3}}\left(b_{3}\right) d b_{3}, & b_{O}>B_{3, \mathrm{~m}} \\
1, & b_{O} \leq B_{3, \mathrm{~m}}
\end{array} .\right.
$$

Similar to the SER, the outage probability for this case will be evaluated numerically because $f_{B_{3}}\left(b_{3}\right)$ (27) does not have a closed-form representation.

\section{Number of Reflectors $L \geq 4$}

In this case, the PDF obtained using the CLT can be used to derive $\bar{P}_{O}$,

$$
\begin{aligned}
\bar{P}_{O} & =\int_{B_{L, \mathrm{~m}}^{2}}^{b_{O}^{2}} f_{Y_{L}}\left(y_{L}\right) d y_{L} \\
& =\frac{1}{\sigma_{Y_{L}} \sqrt{2 \pi}} \int_{B_{L, \mathrm{~m}}^{2}}^{b_{O}^{2}} \exp \left(-\frac{1}{2}\left(\frac{y_{L}-\mu_{Y_{L}}}{\sigma_{Y_{L}}}\right)^{2}\right) d y_{L} \\
& =Q\left(\frac{B_{L, \mathrm{~m}}^{2}-\mu_{Y_{L}}}{\sigma_{Y_{L}}}\right)-Q\left(\frac{b_{O}^{2}-\mu_{Y_{L}}}{\sigma_{Y_{L}}}\right) .
\end{aligned}
$$

\section{NumericAl RESUlts}

This section presents the numerical results obtained from the derived formulae, and compares them to Monte Carlo simulation results using various configurations. The performance of the considered UAVIRS system is evaluated in terms of SER and outage probability. Each simulation point is obtained using $10^{7}$ realizations. The average transmission power for all scenarios is normalized to unity, and the SNR in $\mathrm{dB}$ is defined as $\mathrm{SNR} \triangleq-\log _{10}\left(\sigma_{z}^{2}\right)$. The phase estimates are considered unbiased, i.e., $\mu=0$, and 


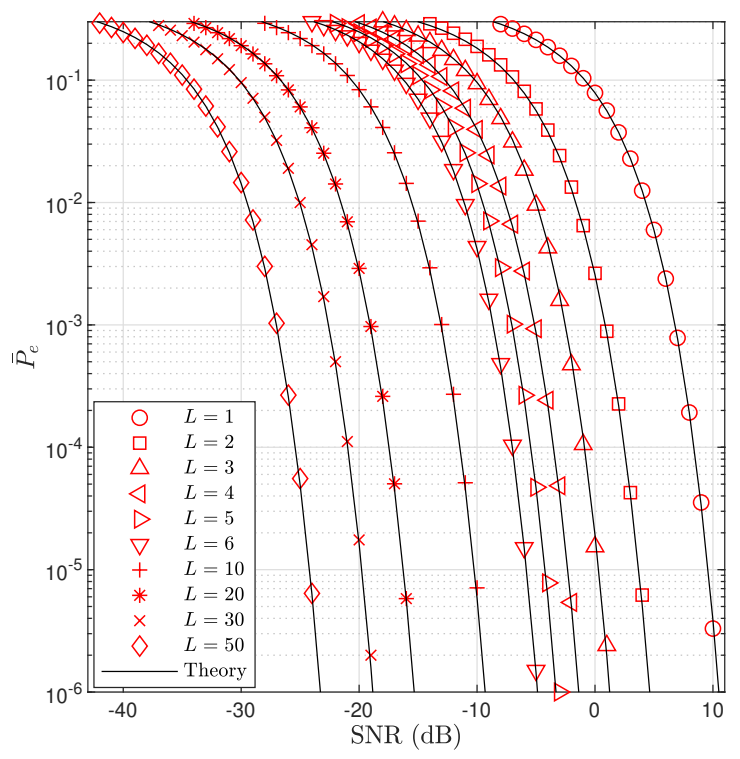

(a) SER

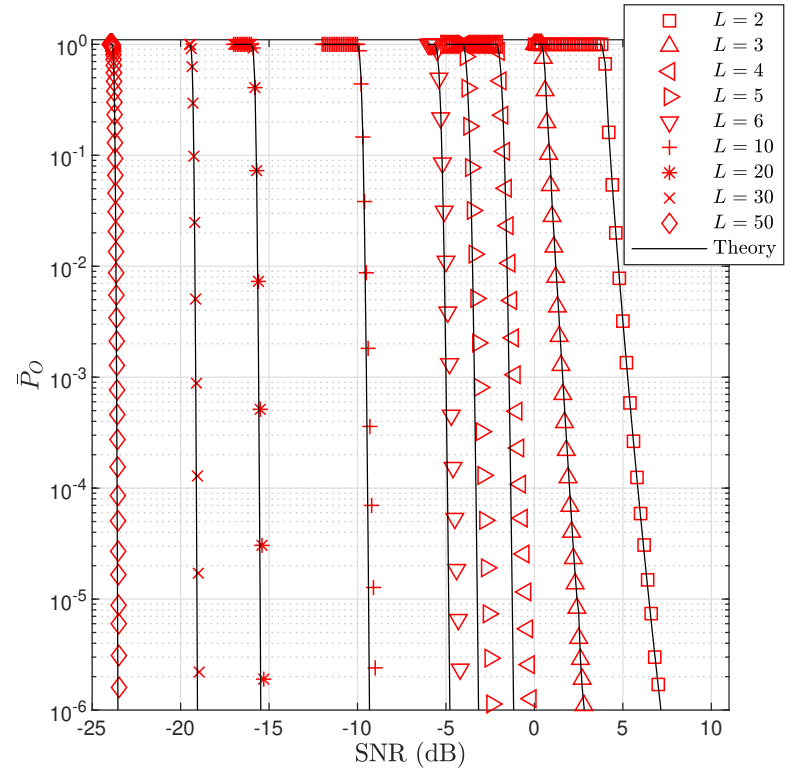

(b) Outage probability

Fig. 3: Analytical and simulated SER and outage probability of the system for various number of reflecting elements $L$, where $\kappa=20$, and $A_{i}=1 \forall i$.

$\kappa_{i}=\kappa \forall i$ is considered for all figures. For the outage probability, the SNR threshold has been set at $\gamma_{O}=10 \mathrm{~dB}$ for all scenarios. The modulation used is binary phase shift keying (BPSK), and hence, the SER and bit error rate (BER) are equal. The analytical SER results for $L=2$ are obtained using (38) and (42) for equal and unequal received signal amplitudes, respectively. On the other hand, the outage probability for $L=2$ is obtained using (54). Unless it is specified otherwise, the SER and outage probabilities for $L=3$ are obtained using (43) and (55), respectively. For $L \geq 4$, the SER is obtained using (46) and the outage probability is obtained using (56). In all infinite summations, we consistently use the first 30 terms.

Fig. 3 shows the analytical and simulated SER and outage probability of the considered system for various values of $L$ where $\kappa=20$, and $A_{i}=1 \forall i$. As can be noted from Fig. 3a, the derived SER expressions match very well the simulation results, including the SER case for $L \geq 4$, which is derived based on the CLT. In addition, the results show the considerable SER enhancement caused by using IRSs. However, the obtained SNR gain decreases as $L$ increases. For example, the SNR gain is about 6 $\mathrm{dB}$ using $L=10$ as compared to $L=20$, while the gain is only $3.5 \mathrm{~dB}$ when increasing $L$ from 20 to 30. The same behavior is obtained for $L=1,2,3$.

Fig. $3 \mathrm{~b}$ shows the outage probability of the system where the SNR threshold $\gamma_{O}=10 \mathrm{~dB}$. According 


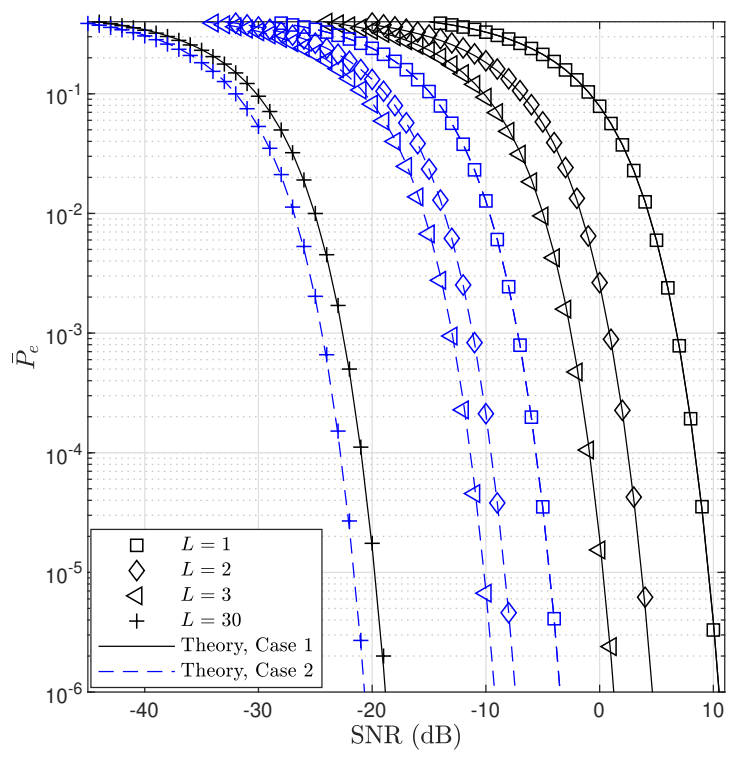

(a) SER

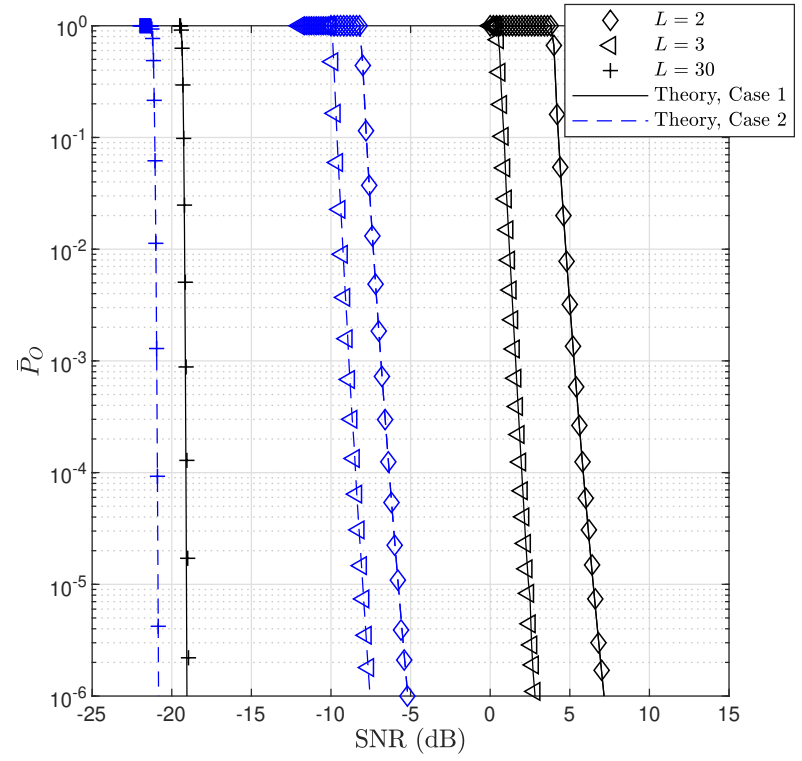

(b) Outage probability

Fig. 4: Analytical and simulated SER and outage probability for various values of $L$ with equal and unequal $A_{i}$, and for $\kappa=20$.

to the figure, a perfect match between simulations and analysis is obtained for $L \leq 3$ and $L \geq 20$. However, for the remaining cases, i.e., $4 \leq L<20$, a small mismatch can be noted when $\bar{P}_{O}$ is below $10^{-3}$. The small difference is due to the CLT, which becomes more accurate by increasing $L$. Moreover, it can be observed that using IRSs can significantly enhance the outage probability. Nevertheless, the outage probability curves are very steep at high values of $L$ because the received signal power distribution is very narrow, and thus, small SNR changes may cause significant change in the outage probability.

Fig. 4 shows the analytical and simulated system SER and outage probability for various values of $L$, and two cases for $A_{i}$. In case $1, A_{i}=1 \forall i$, and in Case $2,\left\{A_{1}, A_{2}, A_{3}\right\}=\{5,3,2\}$ and $A_{4}, A_{5}, \ldots, A_{30}=1$. For all cases in the figure $\kappa=20$. As can be noted from Fig. 4a, the SER analytical results match very well the simulation results for all the considered scenarios. The results are presented for the unequal amplitudes as well to evaluate the impact of the signal amplitude on $\bar{P}_{\mathrm{e}}$. For example, for the case of $L=1$ with $A_{1}=5$ has approximately $5 \mathrm{~dB}$ improvement over the case of $L=3$ with $\left\{A_{1}, A_{2}, A_{3}\right\}=1$. This implies that the link quality has a significant effect on the system performance in addition to the number of reflectors $L$. Fig. $4 \mathrm{~b}$ shows the outage probability. As can be observed from the figure, the obtained analysis perfectly matches the simulation results. The figure also 


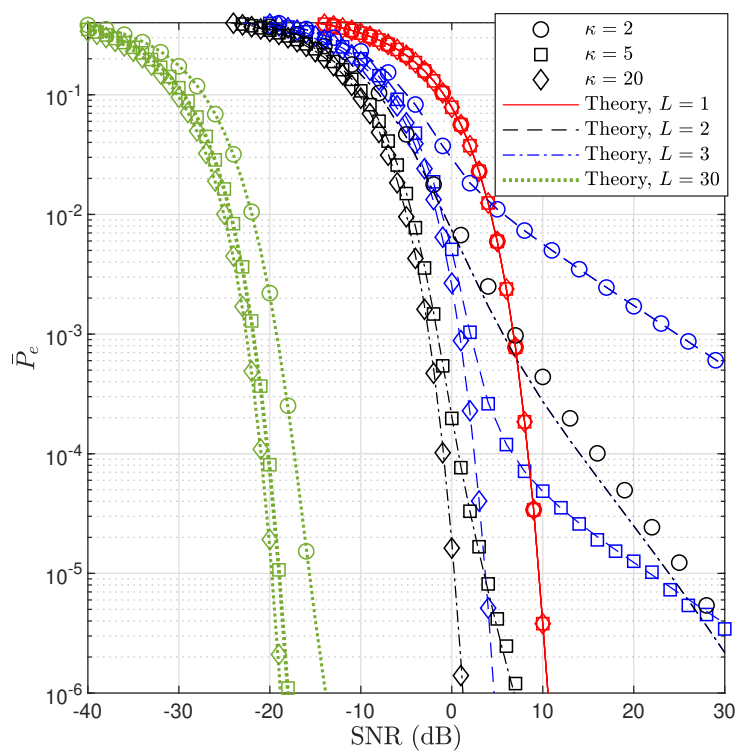

(a) SER

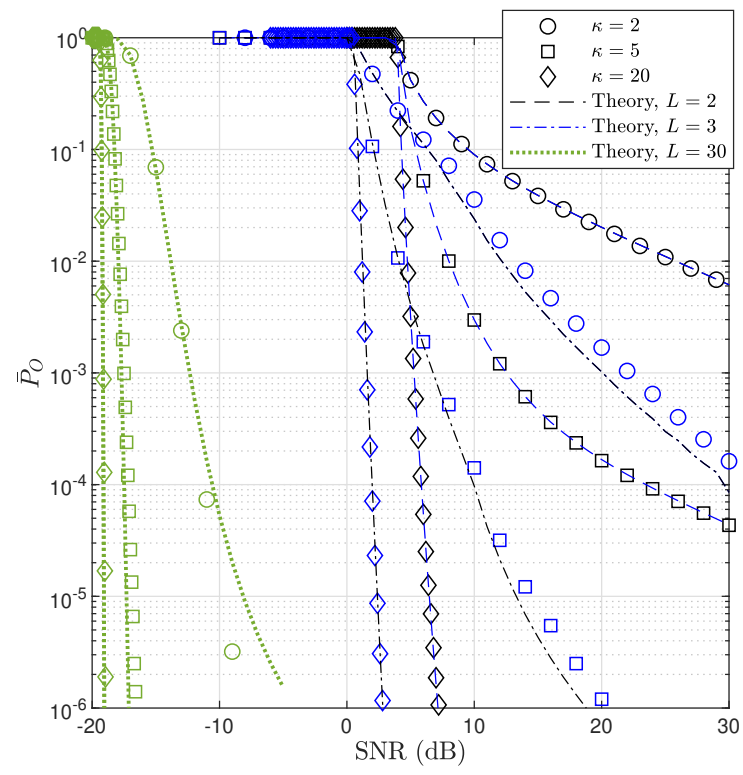

(b) Outage probability

Fig. 5: SER and outage probability for various values of $\kappa$ and $L$ using $A_{i}=1 \forall i$.

shows that the outage probability is inversely proportional to the link quality.

Fig. 5a shows the SER for different values of $\kappa$ and $L$ using $A_{i}=1 \forall i$. The SER of $L=1$ is used as a benchmark, and its is not affected by $\kappa$ since the receiver is assumed to know the overall sgnal phase accurately. For the theoretical results. As can be noted from the figure, the analysis matches the simulation results for all cases except for $L=3$ with $\kappa \leq 5$, where some mismatch is resulted from the multiple discontinuities in $b_{3}^{2}$ that appear at low $\kappa$ values. It can be also noted that large values of $\kappa$ correspond to small phase errors, and thus, better SER. The figure also shows that the SER degradation versus $\kappa$ depends on $L$. For small values of $L$ the SER is very sensitive to the variations of $\kappa$. Therefore, increasing the number of reflectors is an efficient approach to mitigate the phase errors. The same observations and conclusions can be generally made for the outage probability in Fig. $5 \mathrm{~b}$.

Fig. 6 shows the SER and outage probability for various values of $L, A_{i}=1 \forall i$, and $\kappa=5$. All the results in the figure are obtained using the CLT given in (46) and (56) for the SER and outage probability, respectively. As can be noted from Fig. 6a, the simulation results deviate significantly from the theoretical results obtained using CLT when $L<4$. However, the mismatch decreases for $L \geq 4$ and becomes negligible for $L \geq 6$. Therefore, the accurate analysis for the cases of $L=2$ and 3 is necessary to provide accurate analytical results for such cases. The outage probability results in Fig. $6 \mathrm{~b}$ show a higher deviation between the simulation and analytical results obtained suing the CLT, particularly for 


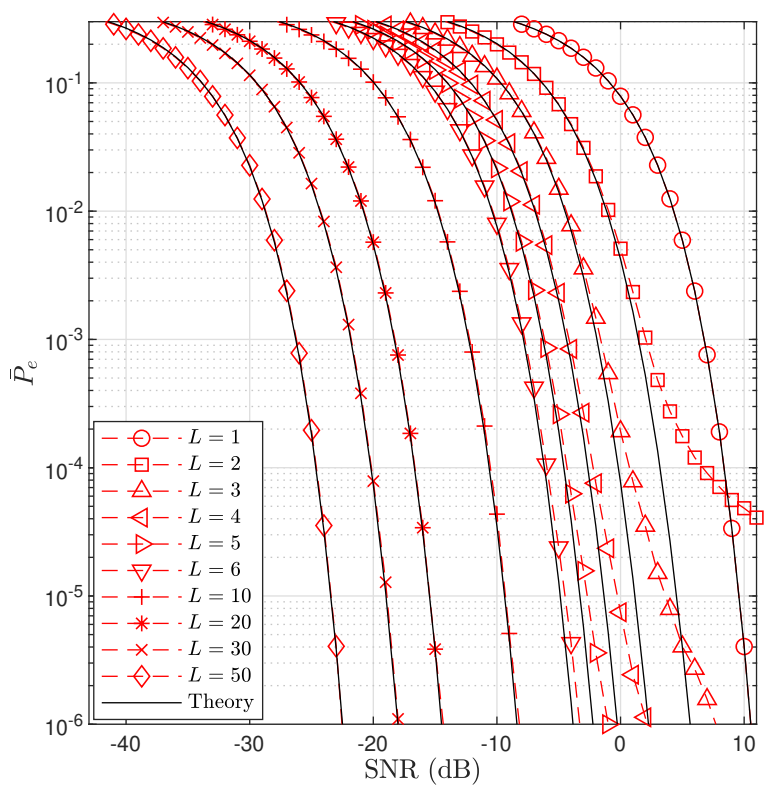

(a) SER

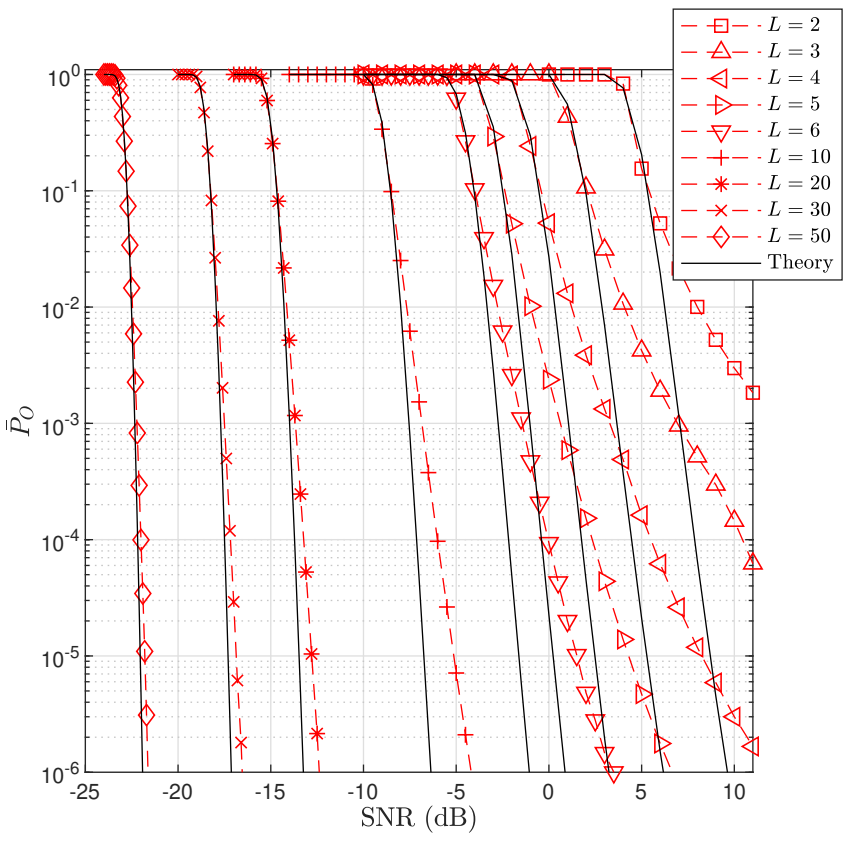

(b) Outage probability

Fig. 6: The SER and outage probability for different values of $L$, where the CLT is applied for all $L$, and $\kappa=5$.

$L<10$. Moreover, it can be noted that the deviation becomes more apparent for $\bar{P}_{O}<10^{-3}$.

Fig. 7 is produced using the same settings of Fig. 6 except that $\kappa=20$. As can be noted from Fig. 7a, the CLT in this case gives near perfect match even for $L=\{1,2,3\}$. Such performance is obtained because at high values of $\kappa$ the PDF of the envelope becomes mostly concentrated around $B_{L, \mathrm{M}}$, and hence, averaging the conditional SER over the PDF will be mostly dependent on the mean and variance of the PDF rather than the actual shape of the PDF. For the outage probability the scenario is different because outage computation involves integration over the PDF itself with no averaging operation. Therefore, it can be noted from the results in Fig. $7 \mathrm{~b}$ the CLT does not provide accurate results for $L<10$.

Fig. 8 shows the SER versus SNR for the cases where the signals have fixed and random amplitudes. For the random amplitudes, the fading factor is modeled as Rician distribution with parameters $\Omega$ and $\mathcal{K}$ i.e., $A_{i} \sim \mathcal{R}(\Omega, \mathcal{K})$. For fair comparison, we set $\Omega=1$ for each of the $L$ signals in the Rician case and $A_{i}=1 \forall i$ for the fixed amplitudes case. As can be noted from the figure, the SER performance for the Rician model converges to the fixed amplitude model when $L$ or $\mathcal{K}$ increases. For example, it can be noted that $\bar{P}_{e}\left|\left(A_{i}=1\right) \approx \bar{P}_{e}\right|\left(A_{i} \sim \mathcal{R}(1,20)\right)$. Moreover, the SNR gain obtained by increasing $\mathcal{K}$ 


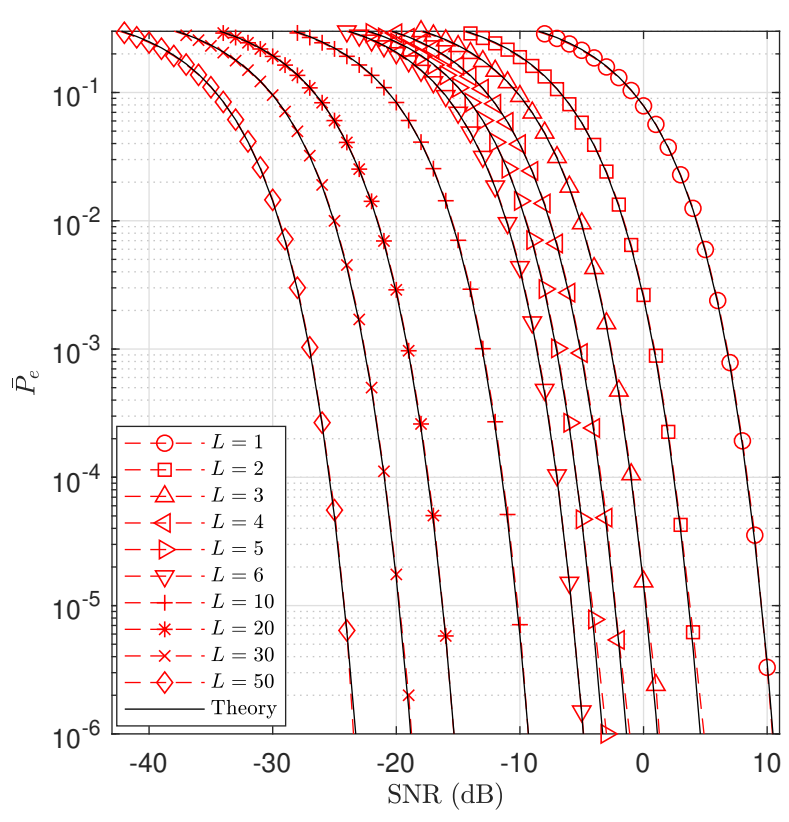

(a) SER

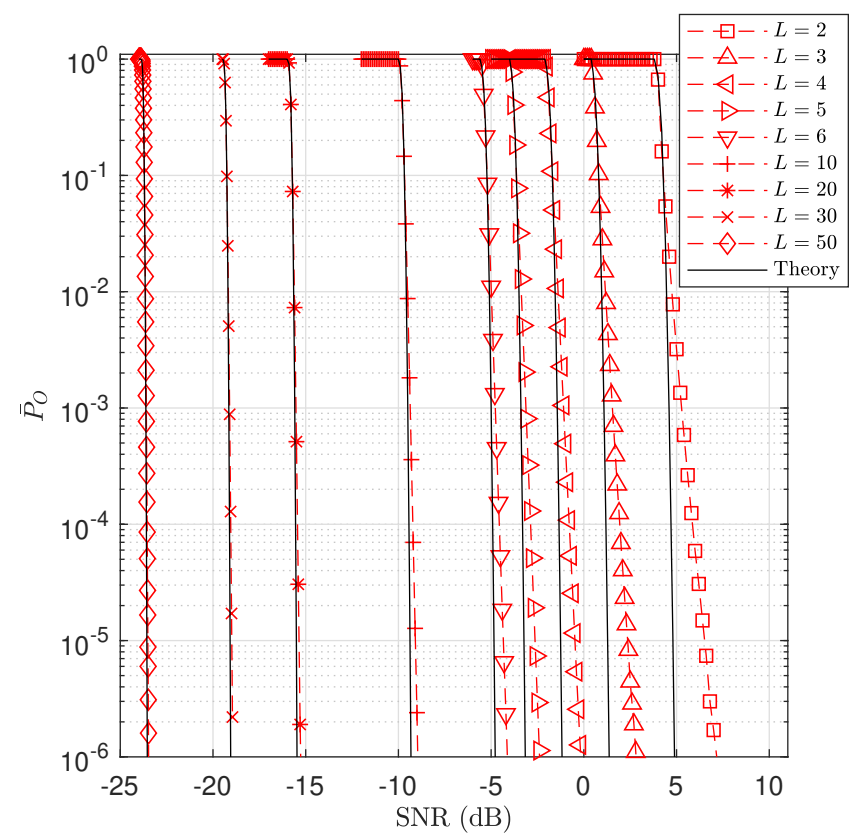

(b) Outage probability

Fig. 7: The SER and outage probability for different values of $L$, where the CLT is applied for all $L$, and $\kappa=20$.

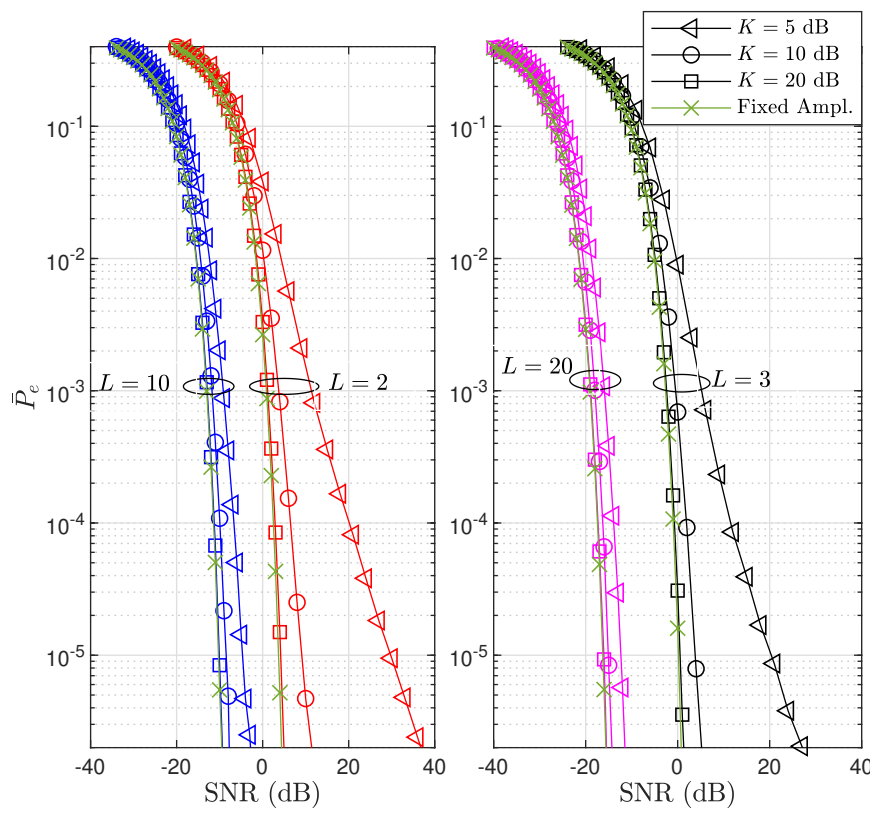

Fig. 8: The SER for Rician distributed amplitudes, i.e., $A_{i} \sim \mathcal{R}(\Omega, \mathcal{K})$. 
becomes less important as $L$ increases. For example, at $\bar{P}_{e}=10^{-5}$, the SNR gain obtained by increasing $\mathcal{K}$ from 5 to $20 \mathrm{~dB}$ is $27 \mathrm{~dB}$ when $L=2$, while it is almost $4 \mathrm{~dB}$ when $L=20$.

\section{CONCLUSION}

The SER and outage performance of IRS assisted UAV-UAV communications were investigated when phase compensation at the reflectors is imperfect. The derivations for the SER and outage probability were provided for $L=\{1,2,3\}$ using SAT, and CLT when $L \geq 4$. The results provided an insight on the interplay between the number of elements, phase errors and system performance. It was demonstrated that IRS significantly improve the performance of UAV-UAV communications, particularly for large values of $L$. More interestingly however, it was shown that increasing the number of reflectors provides some form of immunity against phase error. On the other hand, when $L$ is small, the degradation due to large phase errors may surpass the IRS gain, hence it is paramount that the system designer is aware of the amount of phase error. In addition, the results revealed that the accuracy of the CLT approximation improves as $L$ and $\kappa$ increase. Finally, it was found that the nonfading amplitudes model can be used to accurately model the fading amplitudes with Rician fading given that the Rician factor, or the number of reflectors, is large.

\section{APPENDIX I}

The expected value of $\cos \left(n \phi_{j}\right)$ can be expressed as

$$
\mathrm{E}\left[\cos \left(n \phi_{j}\right)\right]=\frac{1}{2 \pi I_{0}\left(\kappa_{j}\right)} \int_{-\pi}^{\pi} \cos \left(n \phi_{j}\right) \mathrm{e}^{\kappa_{j} \cos \left(\phi_{j}\right)} d \phi_{j}
$$

By dividing the interval of the integral into two subintervals, $[-\pi, 0]$ and $(0, \pi], \mathrm{E}\left[\cos \left(n \phi_{j}\right)\right]$ and substituting $\theta=\phi_{j}$ in the first integral, and noting that $\cos (-\theta)=\cos \theta$ yields

$$
\begin{aligned}
\mathrm{E}\left[\cos \left(n \phi_{j}\right)\right] & =\frac{1}{2 \pi I_{0}\left(\kappa_{j}\right)}\left(-\int_{-\pi}^{0} \cos (n \theta) \mathrm{e}^{\kappa_{j} \cos (\theta)} d \theta+\int_{0}^{\pi} \cos \left(n \phi_{j}\right) \mathrm{e}^{\kappa_{j} \cos \left(\phi_{j}\right)} d \phi_{j}\right) \\
& =\frac{1}{2 \pi I_{0}\left(\kappa_{j}\right)}\left(\int_{0}^{\pi} \cos (n \theta) \mathrm{e}^{\kappa_{j} \cos (\theta)} d \theta+\int_{0}^{\pi} \cos \left(n \phi_{j}\right) \mathrm{e}^{\kappa_{j} \cos \left(\phi_{j}\right)} d \phi_{j}\right) \\
& =\frac{1}{\pi I_{0}\left(\kappa_{j}\right)} \int_{0}^{\pi} \cos \left(n \phi_{j}\right) \mathrm{e}^{\kappa_{j} \cos \left(\phi_{j}\right)} d \phi_{j}
\end{aligned}
$$

Consequently, the definition of the modified Bessel function can be used,

$$
I_{n}(x)=\frac{1}{\pi} \int_{0}^{\pi} \cos (n \theta) \mathrm{e}^{x \cos (\theta)} d \theta
$$


and thus, $\mathrm{E}\left[\cos \left(n \phi_{j}\right)\right]$ can be written as

$$
E\left[\cos \left(n \phi_{j}\right)\right]=\frac{I_{n}\left(\kappa_{j}\right)}{\left.I_{0}\left(\kappa_{j}\right)\right)} .
$$

On the other hand, the expected value of $\cos \left(n \phi_{j}\right)$ can be expressed as

$$
\begin{aligned}
\mathrm{E}\left[\sin \left(n \phi_{j}\right)\right] & =\frac{1}{2 \pi I_{0}\left(\kappa_{j}\right)} \int_{-\pi}^{\pi} \sin \left(n \phi_{j}\right) \mathrm{e}^{\kappa_{j} \cos \left(\phi_{j}\right)} d \phi_{j} \\
& =\frac{1}{2 \pi I_{0}\left(\kappa_{j}\right)}\left(\int_{-\pi}^{0} \sin \left(n \phi_{j}\right) \mathrm{e}^{\kappa_{j} \cos \left(\phi_{j}\right)} d \phi_{j}+\int_{0}^{\pi} \sin \left(n \phi_{j}\right) \mathrm{e}^{\kappa_{j} \cos \left(\phi_{j}\right)} d \phi_{j}\right) \\
& =0
\end{aligned}
$$

where the last equality is obtained by substituting $\theta=-\phi_{j}$ and noting that $\sin (-\theta)=-\sin \theta$ while $\cos (-\theta)=\cos \theta$.

\section{APPENDIX II}

The values of $\mu_{y_{L}}$ and $\sigma_{y_{L}}^{2}$ can be derived as the following

$$
\begin{aligned}
\mu_{y} & =\mathrm{E}\left[B_{L}^{2}\right] \\
& =\mathrm{E} \mid\left[\left.\mathbf{A}\right|^{2}\right]+2 \sum_{L \geq j>k \geq 1} \mathrm{E}\left[A_{j} A_{k} \cos \left(\phi_{j}-\phi_{k}\right)\right] \\
& =\sum_{i=1}^{L} \mathrm{E}\left[A_{i}^{2}\right]+2 \sum_{L \geq j>k \geq 1} \mathrm{E}\left[A_{j}\right] \mathrm{E}\left[A_{k}\right] \mathrm{E}\left[\cos \left(\phi_{j}-\phi_{k}\right)\right]
\end{aligned}
$$

After some mathematical manipulations and given $A_{i} \forall i$ is fixed, then $\mu_{y}$ can be computed as

$$
\begin{aligned}
E\left[Y_{L}\right] & =\sum_{i=1}^{L} A_{i}^{2}+2 \sum_{L \geq j>k \geq 1} A_{j} A_{k}\left(\mathrm{E}\left[\cos \phi_{j}\right] \mathrm{E}\left[\cos \phi_{k}\right]+\mathrm{E}\left[\sin \phi_{j}\right] \mathrm{E}\left[\sin \phi_{k}\right]\right) \\
& =\sum_{i=1}^{L} A_{i}^{2}+2 \sum_{L \geq j>k \geq 1} A_{j} A_{k} \frac{I_{1}\left(\kappa_{j}\right) I_{1}\left(\kappa_{k}\right)}{I_{0}\left(\kappa_{j}\right) I_{0}\left(\kappa_{k}\right)} .
\end{aligned}
$$

The variance $\sigma_{Y_{L}}^{2}$ can be computed as

$$
\sigma_{Y_{L}}^{2} \triangleq \mathrm{E}\left[Y_{L}^{2}\right]-\mathrm{E}^{2}\left[Y_{L}\right]
$$


where

$$
\begin{aligned}
\mathrm{E}\left[Y_{L}^{2}\right] & =\mathrm{E}\left[\left(|\mathbf{A}|^{2}+2 \sum_{L \geq j>k \geq 1} A_{j} A_{k} \cos \left(\phi_{j}-\phi_{k}\right)\right)^{2}\right] \\
& =\left(|\mathbf{A}|^{2}\right)^{2}+\underbrace{4 \mathrm{E}\left[\left(\sum_{L \geq j>k \geq 1} A_{j} A_{k} \cos \left(\phi_{j}-\phi_{k}\right)\right)^{2}\right]}_{T_{1}}+\underbrace{4|\mathbf{A}|^{2} \sum_{L \geq j>k \geq 1} A_{j} A_{k} \mathrm{E}\left[\cos \left(\phi_{j}-\phi_{k}\right)\right]}_{T_{2}}
\end{aligned}
$$

where the term $T_{2}$ can be derived as

$$
\begin{aligned}
T_{2} & =4|\mathbf{A}|^{2} \sum_{L \geq j>k \geq 1} A_{j} A_{k} \mathrm{E}\left[\cos \left(\phi_{j}-\phi_{k}\right)\right] \\
& =4|\mathbf{A}|^{2} \sum_{L \geq j>k \geq 1} A_{j} A_{k} \frac{I_{1}\left(\kappa_{j}\right) I_{1}\left(\kappa_{k}\right)}{I_{0}\left(\kappa_{j}\right) I_{0}\left(\kappa_{k}\right)}
\end{aligned}
$$

The term $T_{1}$ can be derived as

$$
\begin{aligned}
T_{1} & =4 \mathrm{E}\left[\left(\sum_{n \geq j>k \geq 1} A_{j} A_{k} \cos \left(\phi_{j}-\phi_{k}\right)\right)^{2}\right] \\
& =\underbrace{4 \sum_{L \geq j>k \geq 1} A_{j}^{2} A_{k}^{2} \mathrm{E}\left[\cos ^{2}\left(\phi_{j}-\phi_{k}\right)\right]}_{T_{1,1}}+\underbrace{8 \sum_{L \geq j>k \geq 1} \sum_{L \geq i>l>1} A_{j} A_{k} A_{i} A_{l} \mathrm{E}\left[\cos \left(\phi_{j}-\phi_{k}\right)\right] \mathrm{E}\left[\cos \left(\phi_{i}-\phi_{l}\right)\right]}_{T_{1,2}}
\end{aligned}
$$

The term $T_{1,1}$ is given by

$$
\begin{aligned}
T_{1,1} & =4 \sum_{L \geq j>k \geq 1} A_{j}^{2} A_{k}^{2} \mathrm{E}\left[\cos ^{2}\left(\phi_{j}-\phi_{k}\right)\right] \\
& =2 \sum_{L \geq j>k \geq 1} A_{j}^{2} A_{k}^{2}\left(1+\mathrm{E}\left[\cos \left(2 \phi_{j}-2 \phi_{k}\right)\right]\right) \\
& =2 \sum_{L \geq j>k \geq 1} A_{j}^{2} A_{k}^{2}\left(1+\mathrm{E}\left[\cos \left(2 \phi_{j}\right) \cos \left(2 \phi_{k}\right)+\sin \left(2 \phi_{j}\right) \sin \left(2 \phi_{k}\right)\right]\right) \\
& =2 \sum_{L \geq j>k \geq 1} A_{j}^{2} A_{k}^{2}\left(1+\frac{I_{2}\left(\kappa_{j}\right)}{I_{0}\left(\kappa_{j}\right)} \frac{I_{2}\left(\kappa_{k}\right)}{I_{0}\left(\kappa_{k}\right)}\right) .
\end{aligned}
$$


The term $T_{1,2}$ is

$$
\begin{aligned}
T_{1,2} & =8 \sum_{L \geq j>k \geq 1} \sum_{L \geq i>l>1} A_{j} A_{k} A_{i} A_{l} \mathrm{E}\left[\cos \left(\phi_{j}-\phi_{k}\right)\right] \mathrm{E}\left[\cos \left(\phi_{i}-\phi_{l}\right)\right] \\
& =8 \sum_{L \geq j>k \geq 1} \sum_{L \geq i>l>1} A_{j} A_{k} A_{i} A_{l} \frac{I_{1}\left(\kappa_{j}\right) I_{1}\left(\kappa_{k}\right) I_{1}\left(\kappa_{i}\right) I_{1}\left(\kappa_{l}\right)}{I_{0}\left(\kappa_{j}\right) I_{0}\left(\kappa_{k}\right) I_{0}\left(\kappa_{i}\right) I_{0}\left(\kappa_{l}\right)}
\end{aligned}
$$

\section{REFERENCES}

[1] Q. Wu and R. Zhang, "Intelligent reflecting surface enhanced wireless network via joint active and passive beamforming," IEEE Trans. Wireless Commun., vol. 18, no. 11, pp. 5394-5409, Nov. 2019.

[2] Q. Wu and R. Zhang, "Towards smart and reconfigurable environment: Intelligent reflecting surface aided wireless network," IEEE Commun. Mag., vol. 58, no. 1, pp. 106-112, Jan. 2020.

[3] H. Xie, J. Xu, and Y. Liu, "Max-min fairness in IRS-aided multi-cell MISO systems via joint transmit and reflective beamforming," ICC 2020 - 2020 IEEE Int. Conf. Commun. (ICC), Dublin, Ireland, 2020, pp. 1-6.

[4] Y. Tang, G. Ma, H. Xie, J. Xu, and X. Han, "Joint transmit and reflective beamforming design for IRS-assisted multiuser MISO SWIPT systems," ICC 2020 - 2020 IEEE Int. Conf. Commun. (ICC), Dublin, Ireland, 2020, pp. 1-6.

[5] D. Xu, X. Yu, Y. Sun, D. Ng, and R. Schober, "Resource allocation for IRS-assisted full-duplex cognitive radio systems," IEEE Tran. Commun., to be published. doi: 10.1109/TCOMM.2020.3020838.

[6] Y. Gao, J. Xu, W. Xu, D. Ng, and M.-S. Alouini, "Distributed IRS with statistical passive beamforming for MISO communications," IEEE Wireless Commun. Lett., to be published. doi: 10.1109/LWC.2020.3024952.

[7] X. Yu, D. Xu, Y. Sun, D. Ng, and R. Schober, "Robust and secure wireless communications via intelligent reflecting surfaces," IEEE J. Sel. Areas Commun., to be published. doi: 10.1109/JSAC.2020.3007043.

[8] G. Yu, X. Chen, C. Zhong, D. Ng, and Z. Zhang, "Design, analysis, and optimization of a large intelligent reflecting surfaceaided B5G cellular internet of things," IEEE Internet Things J., vol. 7, no. 9, pp. 8902-8916, Sep. 2020.

[9] J. Zuo, Y. Liu, Z. Qin, and N. Al-Dhahir, "Resource allocation in intelligent reflecting surface assisted NOMA systems," IEEE Trans. Commun., to be published. doi: 10.1109/TCOMM.2020.3016742.

[10] X. Mu, Y. Liu, L. Guo, J. Lin, and N. Al-Dhahir, "Exploiting intelligent reflecting surfaces in NOMA networks: Joint beamforming optimization," IEEE Trans. Wireless Commun., to be published. doi: 10.1109/TWC.2020.3006915.

[11] R. Liu, H. Li, M. Li, and Q. Liu, "Symbol-level precoding design for IRS-assisted MU-MISO systems," 2020 IEEE Wireless Commun. Netw. Conf. (WCNC), Seoul, Korea (South), 2020, pp. 1-6.

[12] H. Lu, Y. Zeng, S. Jin, and R. Zhang, "Enabling panoramic full-angle reflection via aerial intelligent reflecting surface," 2020 IEEE Int. Conf. Commun. Workshops (ICC Workshops), Dublin, Ireland, 2020, pp. 1-6.

[13] S. Abeywickrama, R. Zhang, Q. Wu, and C. Yuen, "Intelligent reflecting surface: Practical phase shift model and beamforming Optimization,” IEEE Trans. Commun., vol. 68, no. 9, pp. 5849-5863, Sep. 2020.

[14] Y. Zeng and R. Zhang, "Energy-efficient UAV communication with trajectory optimization," IEEE Trans. Wireless Commun., vol. 16, no. 6, pp. 3747-3760, Jun. 2017.

[15] A. Fotouhi et al., "Survey on UAV cellular communications: Practical aspects, standardization advancements, regulation, and security challenges," IEEE Commun. Surveys Tuts., vol. 21, no. 4, pp. 3417-3442, Fourthquarter 2019.

[16] X. Wang and M. C. Gursoy, "Coverage analysis for energy-harvesting UAV-assisted mmwave cellular networks," IEEE J. Sel. Areas Commun., vol. 37, no. 12, pp. 2832-2850, 2019.

[17] Y. Zeng, Q. Wu, and R. Zhang, "Accessing from the sky: A tutorial on UAV communications for 5G and beyond," Proc. IEEE, vol. 107, no. 12, pp. 2327-2375, Dec. 2019.

[18] L. Zhu, J. Zhang, Z. Xiao, X. Cao, X.-G. Xia, and R. Schober, "Millimeter-wave full-duplex UAV relay: Joint positioning, beamforming, and power control,” IEEE J. Sel. Areas Commun., vol. 38, no. 9, pp. 2057-2073, Sep. 2020.

[19] S. Khairy, P. Balaprakash, L.Cai, and Y. Cheng, "Constrained deep reinforcement learning for energy sustainable multi-UAV based random access IoT networks with NOMA,” IEEE J. Sel. Areas Commun., IEEE Early Access, 2020.

[20] C. Liu, W. Feng, Y. Chen, C.-X. Wang, and N. Ge, "Cell-free satellite-UAV networks for 6G wide-area internet of things," IEEE J. Sel. Areas Commun., IEEE Early Access, 2020. 
[21] Z. Ding, R. Schober, and H. V. Poor, "On the impact of phase shifting designs on IRS-NOMA," IEEE Wireless Commun. Lett., vol. 9, no. 10, pp. 1596-1600, Oct. 2020.

[22] C. You, B. Zheng, and R. Zhang, "Channel estimation and passive beamforming for intelligent reflecting surface: Discrete phase shift and progressive refinement," IEEE J. Sel. Areas Commun., to be published. doi: 10.1109/JSAC.2020.3007056.

[23] X. Hu, C. Zhong, M.-S. Alouini, and Z. Zhang, "Robust design for IRS-aided communication systems with user location uncertainty," IEEE Wireless Commun. Lett., to be published. doi: 10.1109/LWC.2020.3020850.

[24] X. Dongfang et al., "Resource allocation for IRS-assisted full-duplex cognitive radio systems," arXiv preprint arXiv:2003.07467, 2020.

[25] V. Jamali et al., "Power efficiency, overhead, and complexity tradeoff in IRS-Assisted communications-quadratic-phase-shift design," arXiv preprint arXiv:2009.05956, 2020.

[26] Z. Beixiong and R. Zhang, "Intelligent reflecting surface-enhanced OFDM: Channel estimation and reflection optimization." IEEE Wireless Commun. Lett., 9.4 (2019): 518-522.

[27] H. Alwazani et al., "Intelligent reflecting surface-assisted multi-user MISO communication: Channel estimation and beamforming design.” IEEE Open J. Commun. Soc. 1 (2020): 661-680.

[28] P. Wang et al., "Compressed channel estimation for intelligent reflecting surface-assisted millimeter wave systems," IEEE Signa Process. Lett., (2020).

[29] Z. Wan, Z. Gao Z, and M.-S. Alouini, "Broadband channel estimation for intelligent reflecting surface aided mmWave massive MIMO systems," arXiv preprint arXiv:2002.01629, Feb. 2020.

[30] S. Jiao, F. Fang, X. Zhou, and H. Zhang, "Joint beamforming and phase shift design in downlink UAV networks with IRSassisted NOMA,” J. Commun. Inf. Netw., vol. 5, no. 2, pp. 138-149, June 2020.

[31] D. Ma, M. Ding, and M. Hassan, "Enhancing cellular communications for UAVs via intelligent reflective surface," 2020 IEEE Wireless Commun. Netw. Conf. (WCNC), Seoul, Korea (South), 2020, pp. 1-6.

[32] L. Ge, P. Dong, H. Zhang, J. Wang, and X. You, "Joint beamforming and trajectory optimization for intelligent reflecting surfaces-assisted UAV communications," IEEE Access, vol. 8, pp. 78702-78712, 2020.

[33] Z. Mohamed and S. Aïssa, "Leveraging UAVs with intelligent reflecting surfaces for energy-efficient communications with cell-edge users," 2020 IEEE Int. Conf. Commun. Workshops (ICC Workshops), Dublin, Ireland, 2020, pp. 1-6.

[34] Y. Cai, Z. Wei, S. Hu, D. Ng, and J. Yuan, "Resource allocation for power-efficient IRS-assisted UAV communications," 2020 IEEE Int. Conf. Commun. Workshops (ICC Workshops), Dublin, Ireland, 2020, pp. 1-7.

[35] M. Hua, L. YangL, Q. Wu, C. Pan, C. Li, and A. Swindlehurst, "UAV-assisted intelligent reflecting surface symbiotic radio system," arXiv preprint arXiv:2007.14029, Jul. 2020.

[36] W. Zhiqiang, C. Yuanxin, S. Zhuo, N. Kwan, and Y. Jinhong, "Sum-rate maximization for IRS-assisted UAV OFDMA communication systems," arXiv preprint arXiv:2008.09939, Aug. 2020.

[37] T. Shafique, H. Tabassum, and E. Hossain, "Optimization of wireless relaying with flexible UAV-borne reflecting surfaces," arXiv preprint arXiv:2006.10969, Jun. 2020.

[38] Q. Zhang, W. Saad, and M. Bennis, "Reflections in the sky: Millimeter wave communication with UAV-carried intelligent reflectors," 2019 IEEE Global Commun. Conf. (GLOBECOM) 2019 Dec. 9 (pp. 1-6).

[39] S. Li, B. Duo, X. Yuan, Y. Liang, and M. Di Renzo, "Reconfigurable intelligent surface assisted UAV communication: Joint trajectory design and passive beamforming," IEEE Wireless Commun. Lett., vol. 9, no. 5, pp. 716-720, May 2020.

[40] M. Banagar, H. Dhillon, and A. Molisch, "Impact of UAV wobbling on the air-to-ground wireless channel," IEEE Trans. Veh. Technol., to be published. doi: 10.1109/TVT.2020.3026975

[41] M. Al-Jarrah, A. Al-Dweik, E. Alsusa, and M.-S. Alouni, "Performance analysis of wireless mesh backhauling using intelligent reflecting surface,” TechRxiv. Preprint. [Online]. Available: https://doi.org/10.36227/techrxiv.12543167.v1.

[42] J. Ye, S. Guo, and M.-S. Alouini, "Joint reflecting and precoding designs for SER minimization in reconfigurable intelligent surfaces assisted MIMO systems," IEEE Trans. Wireless Commun., vol. 19, no. 8, pp. 5561-5574, Aug. 2020.

[43] L. Yang, F. Meng, Q. Wu, D. da Costa, and M.-S. Alouini, "Accurate closed-form approximations to channel distributions of RIS-aided wireless systems," IEEE Wireless Commun. Lett., to be published. doi: 10.1109/LWC.2020.3010512.

[44] V. Thirumavalavan and T. Jayaraman, "BER analysis of reconfigurable intelligent surface assisted downlink power domain NOMA system,” 2020 Int. Conf. Commun. Syst. Netw. (COMSNETS), Bengaluru, India, 2020, pp. 519-522. 
[45] A. Boulogeorgos and A. Alexiou, "Performance analysis of reconfigurable intelligent surface-assisted wireless systems and comparison with relaying," IEEE Access, vol. 8, pp. 94463-94483, 2020.

[46] E. Basar, M. Di Renzo, J. De Rosny, M. Debbah, M.-S. Alouini, and R. Zhang, "Wireless communications through reconfigurable intelligent surfaces," IEEE Access, vol. 7, pp. 116753-116773, 2019.

[47] R. Ferreira, M. Facina, F. De Figueiredo, G. Fraidenraich, and E. De Lima, "Bit error probability for large intelligent surfaces under double-Nakagami fading channels," IEEE Open J. Commun. Soc., vol. 1, pp. 750-759, 2020.

[48] Y. Maghsoodi and A. Al-Dweik, "Exact formulae for error-rate analysis of synchronous FHSS networks using envelope characteristic functions of sums of stochastic signals," IEEE Trans. Veh. Technol., vol. 57, pp. 974-985, Mar. 2008.

[49] Y. Maghsoodi and A. Al-Dweik, "Exact amplitude distributions of sums of stochastic sinusoidals and their application in bit error rate analysis,"arXiv preprint arXiv:1911.04746, 2019.

[50] A. Al-Dweik, B. Sharif, and C. Tsimenidis, "Accurate BER analysis of OFDM systems over static frequency-selective multipath fading channels," IEEE Trans. Broadcast., vol. 57, no. 4, pp. 895-901, Dec. 2011.

[51] N. Goddemeier and C. Wietfeld, "Investigation of air-to-air channel characteristics and a UAV specific extension to the Rice model," 2015 IEEE Globecom Workshops (GC Wkshps), San Diego, CA, 2015, pp. 1-5.

[52] B. Du, R. Xue, L. Zhao, and V. Leung, "Coalitional graph game for air-to-air and air-to-ground cognitive spectrum sharing," IEEE Trans. Aerosp. Electron. Syst., vol. 56, no. 4, pp. 2959-2977, Aug. 2020.

[53] F. Ono, T. Kagawa, H. Tsuji, R. Miura, and F. Kojima, "Measurements on C-band air-to-air channel for coexistence among multiple unmanned aircraft systems," 2017 International Conference on Unmanned Aircraft Systems (ICUAS), Miami, FL, USA, 2017, pp. 1160-1164.

[54] K. Takizawa, F. Ono, H. Tsuji, and R. Miura, "Air-to-air radio channel measurement at $2.3 \mathrm{GHz}$ for unmanned aircraft services," 2013 16th International Symposium on Wireless Personal Multimedia Communications (WPMC), Atlantic City, NJ, 2013, pp. 1-5.

[55] C. Yan, L. Fu, J. Zhang, and J. Wang, "A comprehensive survey on UAV communication channel modeling," IEEE Access, vol. 7, pp. 107769-107792, 2019.

[56] K. Takizawa, F. Ono, M. Suzuki, H. Tsuji, and R. Miura, "Measurement on S-band radio propagation characteristics for unmanned aircraft systems," The 8th European Conf. Antennas and Propag. (EuCAP 2014), The Hague, 2014, pp. 3068-3072.

[57] E. Haas, “Aeronautical channel modeling," IEEE Trans. Veh. Technol., vol. 51, no. 2, pp. 254-264, March 2002.

[58] A. Al-Dweik and B. Sharif, "Exact performance analysis of synchronous FH-MFSK wireless networks," IEEE Trans. Veh. Technol., vol. 58, no. 7, pp. 3771-3776, Sept. 2009.

[59] A. Al-Dweik and A. Shami, "Multitone jamming rejection of frequency hopped OFDM systems in wireless channels," 2012 IEEE Veh. Technol. Conf. (VTC Fall), Quebec City, QC, 2012, pp. 1-6.

[60] A. Saci, A. Al-Dweik, A. Shami, and Y. Iraqi, "One-shot blind channel estimation for OFDM systems over frequency-selective fading channels," IEEE Trans. Commun., vol. 65, no. 12, pp. 5445-5458, Dec. 2017.

[61] M. Al-Jarrah, K.-H. Park, A. Al-Dweik, and M.-S. Alouni,"Error rate analysis of amplitude-coherent detection over Rician fading channels with receiver diversity," IEEE Trans. Wireless Commun., vol. 19, no. 1, pp. 134-147, 2020.

[62] I. Gradshteyn and I. Ryzhik, Table of integrals, series, and products. 7th Ed., Academic Press, USA, 2007.

[63] J. Burkardt. "The truncated normal distribution.” Department of Scientific Computing Website, Florida State University, 2014.

[64] A. Goldsmith, Wireless Communications. Cambridge University Press, 2005.

[65] A. Prudnikov, Y. Brychkov, and O. Marichev, Integrals and Series: Special Functions. Gordon and Breach Science Publishers, UK, 3rd edition, 1986.

[66] I. Tanash and T. Riihonen, "Global minimax approximations and bounds for the Gaussian Q-function by sums of exponentials," IEEE Trans. Commun., IEEE Early Access, Jul. 2020. doi: 10.1109/TCOMM.2020.3006902.

[67] A. Prudnikov, Y. Brychkov, and O. Marichev, Integrals and Series: Elementary Functions. Gordon and Breach Science Publishers, UK, 3rd edition, 1986.

[68] Y. Isukapalli and B. Rao, "An analytically tractable approximation for the Gaussian Q-function," IEEE Commun. Lett., vol. 12, no. 9, pp. 669-671, Sep. 2008. 